\title{
Guinea: First Review Under the Three-Year Arrangement Under the Poverty Reduction and Growth Facility, Request for Waiver of Nonobservance of Performance Criteria, Modification of Performance Criteria, Augmentation of Access, and Financing Assurances Review-Staff Report; Staff Statement; Press Release on the Executive Board Discussion; and Statement by the Executive Director for Guinea
}

In the context of first review under the three-year arrangement under the Poverty Reduction and Growth Facility, request for a waiver of nonobservance of performance criteria, modification of performance criteria, augmentation of access, and financing assurances review, the following documents have been released and are included in this package:

- $\quad$ The staff report for First Review Under the Three-Year Arrangement Under the Poverty Reduction and Growth Facility, Request for Waiver of Nonobservance of Performance Criteria, Modification of Performance Criteria, Augmentation of Access, and Financing Assurances Review, prepared by a staff team of the IMF, following discussions that ended on April 2, 2008, with the officials of Guinea on economic developments and policies. Based on information available at the time of these discussions, the staff report was completed on June 30, 2008. The views expressed in the staff report are those of the staff team and do not necessarily reflect the views of the Executive Board of the IMF.

- $\quad$ A staff statement of July 28, 2008 updating information on recent developments.

- $\quad$ A Press Release summarizing the views of the Executive Board as expressed during its July 28, 2008 discussion of the staff report that completed the review.

- $\quad$ A statement by the Executive Director for Guinea.

The documents listed below have been or will be separately released.

Letter of Intent sent to the IMF by the authorities of Guinea* Amendments to the Technical Memorandum of Understanding*

*Also included in Staff Report

The policy of publication of staff reports and other documents allows for the deletion of market-sensitive information.

\author{
Copies of this report are available to the public from \\ International Monetary Fund • Publication Services \\ $70019^{\text {th }}$ Street, N.W. • Washington, D.C. 20431 \\ Telephone: (202) 623-7430 • Telefax: (202) 623-7201 \\ E-mail: publications@imf.org • Internet: http://www.imf.org
}

Price: $\$ 18.00$ a copy

\section{International Monetary Fund Washington, D.C.}





\title{
INTERNATIONAL MONETARY FUND
}

GUINEA

\section{First Review Under the Three-Year Arrangement Under the Poverty Reduction and Growth Facility, Request for Waiver of Nonobservance of Performance Criteria, Modification of Performance Criteria, Augmentation of Access, and Financing Assurances Review}

\author{
Prepared by the African Department \\ (in consultation with other departments) \\ Approved by Robert Sharer and Mark Plant
}

June 30, 2008

\section{Program review}

Staff recommends completion of the first review under the PRGF arrangement based on Guinea's performance and on understandings reached on policy implementation for the remainder of 2008.

All but two quantitative performance criteria (PCs) were met. Staff supports the authorities' requests for waivers of nonobservance, based on their remedial actions. Progress of structural reforms was broadly satisfactory and all structural PCs and benchmarks for end-December 2007 were met. However, several quantitative indicative targets for end-March 2008 were missed, in part on account of a delayed response to the financial pressures arising from higher fuel prices. Actions to meet several structural benchmarks scheduled for early 2008 have been delayed, and two structural PCs for end-June 2008 will be missed.

Given the shock on fuel and food prices and the authorities' broadly appropriate policy response, staff supports the requested modification of several PCs for end-June 2008, and an augmented access to PRGF resources equivalent to 20 percent of quota.

A revised letter of intent describing the economic and financial policies for 2008 is attached to the report.

\section{Participants}

The mission (March 20-April 2, 2008) comprised Messrs. Le Dem (head), Geiregat, Gorbanyov, and Wane, Ms. Qureshi (all AFR), and Mr. Hilaire (resident representative). Mr. Bah (OED) participated in policy discussions, which continued in Washington during the Spring meetings. The mission worked closely with overlapping missions from the World Bank and the AfDB.

The mission met with then Prime Minister Kouyaté; Minister of the Economy, Finance, and Planning Doré; Governor of the Central Bank Bangoura; other officials; and representatives of donors, the private sector, civil society organizations, and the media. 


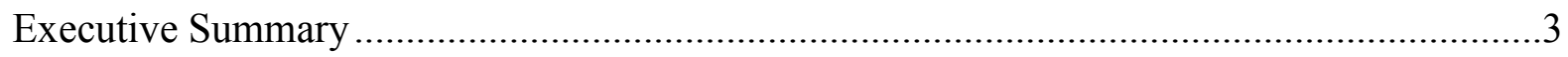

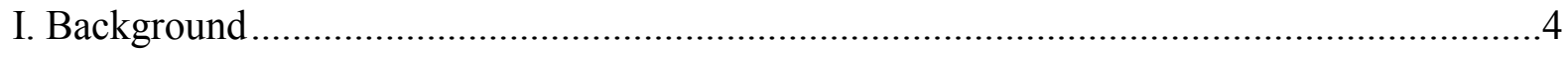

II. Recent Economic Performance ............................................................................4

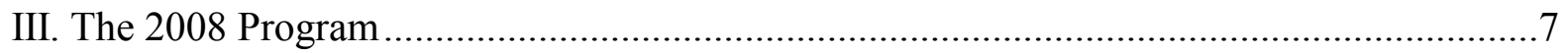

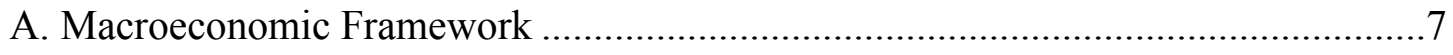

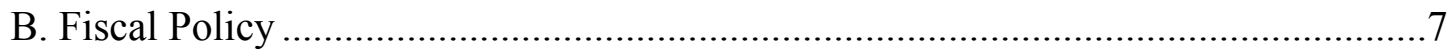

C. Monetary and Exchange Rate Policies .............................................................. 9

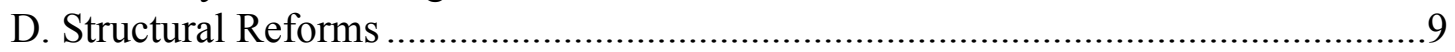

E. External Policies and Outlook ................................................................... 10

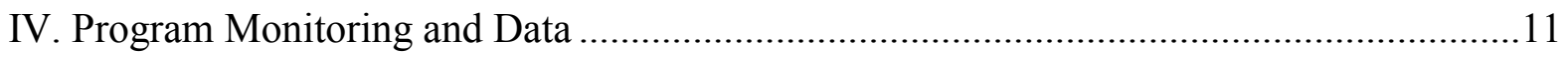

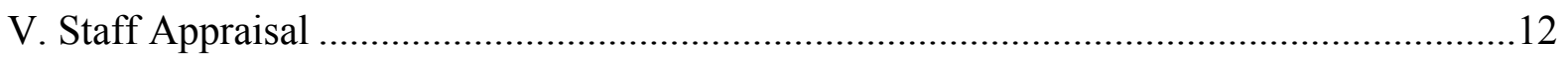

Tables

1. Selected Economic and Financial Indicators, 2005-10 .......Error! Bookmark not defined.

2. Financial Operations of the Central Government, 2005-10 Error! Bookmark not defined.

3. Financial Operations of the Central Government, 2005-10 Error! Bookmark not defined.

4. Monetary Survey, 2006-08 ............................................Error! Bookmark not defined.

5. Balance of Payments, 2005-13 .......................................Error! Bookmark not defined.

6. Indicators of Capacity to Repay the Fund, 2005-16 ............Error! Bookmark not defined.

7. Millennium Development Goals, 1990-2015 .....................Error! Bookmark not defined.

8. Guinea: Disbursements Under the PRGF Arrangement, 2007-10 .................................22

Figure

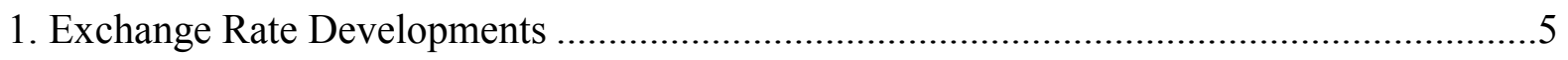

Appendix

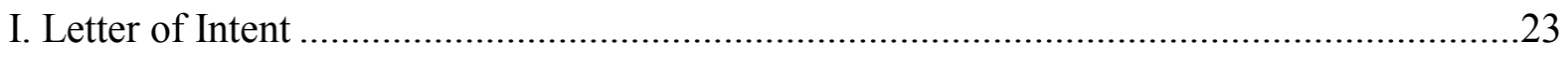

Attachment I. Amendments to the Technical Memorandum of Understanding (TMU)..........33

Annexes

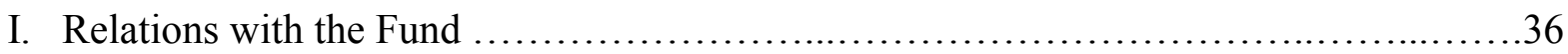

II. Relations with the World Bank .............................................42

III. Statistical Issues ..................................................... 44

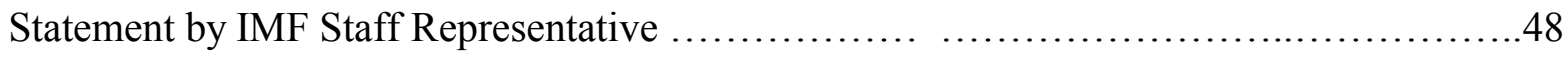

Press Release .............................................................49

Statement by Mr. Rutayisire, Executive Director for Guinea .........................51 


\section{EXECUTIVE SUMMARY}

Guinea has continued to experience social and political instability, compounded by the impact of the world food and fuel price increases. Following the dismissal of the Prime Minister and subsequent military unrest, the authorities agreed to pay wage arrears to soldiers. They also postponed the implementation of the petroleum price adjustment formula (thereby missing an end-June 2008 structural performance criterion (SPC)), stressing acute social risks tied to increases in pump prices on top of the 63 percent adjustment on April $1^{\text {st }} 2008$.

Economic stabilization policies in 2007 were broadly successful. Fiscal and monetary discipline helped stem inflation, and economic activity made a timid recovery. Fiscal targets were broadly achieved, in spite of a revenue shortfall, and the absence of central bank financing of the government kept monetary expansion under control.

\section{However, two quantitative performance criteria (PCs) for end-December 2007 and} several indicative targets for end-March 2008 were missed. Net international reserves did not build up as expected at end-2007, leading to external arrears to some multilateral creditors. These arrears were settled by end-March 2008, and, since then, foreign exchange interventions brought the central bank back to a path of reserves accumulation. The endMarch fiscal target was missed because of financial pressures arising from higher fuel prices.

\section{Policy discussions focused on the best response to the worsened prospects in 2008.}

- $\quad$ Fiscal policy. The authorities are implementing additional tax administration measures and cutting expenditures. Nevertheless, the target for the basic primary balance had to be slightly reduced to make room for measures to cushion the impact of higher food and petroleum prices. These are expected to be partly financed by donors. Fiscal exposure to the public electricity company has been limited.

- Monetary and exchange rate policies. The central bank intends to bring official reserves to one month of import cover by year-end, as originally programmed, but the intra-annual path for accumulation has been modified. Planning of, and monitoring and reporting on debt service payments and reserve accumulation will be strengthened. The monetary program seeks to limit annual inflation to 15 percent.

- Structural reforms. Although central bank reforms are expected to continue at a fair pace and in line with recommendations of the safeguards assessment report, the new central bank law is expected to be submitted with a five-month delay (missing the end-June 2008 SPC). A multiyear action plan to reform public finance management will be adopted by end-June 2008, and mining sector reforms will be accelerated.

- External policies. The decree banning agricultural exports has not been renewed. Guinea is seeking additional financial assistance to address the negative short-term impact of price shocks to the balance of payments, including through a request for augmentation of access to PRGF resources of 20 percent of quota. 


\section{BACKGROUND}

1. Guinea has continued to experience social and political instability, which has been compounded by the impact of the world food and fuel price increases. The threat of a general strike led the government to concede significant mitigating measures for civil servants after petroleum prices were increased by 63 percent in April 2008. Mounting tensions between President Conté and Prime Minister Kouyaté (whom he had appointed to appease civil protests in early 2007) wound up with the dismissal of the latter on May 20, 2008. Following subsequent unrest in the military, the authorities accommodated the repayment of wage arrears to soldiers and decided to postpone the implementation of the petroleum price adjustment formula until end-November 2008. Although the authorities have requested the modification of several program targets to take into account these developments, they remain committed to the program.

\section{Recent ECONOMic Performance}

2. Economic stabilization policies were broadly successful in 2007. Fiscal and monetary discipline helped stem inflation. The nominal effective exchange rate ended the year about one third stronger, the premium on the parallel market declined to about

1 percent

(Text Table 1

and Figure 1),

and exchange

rate volatility

eased. CPI

inflation

dropped by two-

thirds from

\begin{tabular}{|lrrrrr|}
\hline \multicolumn{5}{|c|}{ Text Table 1. Selected Economic Indicators, 2005-07 } \\
\hline & 2005 & 2006 & 2007 & \\
\cline { 2 - 5 } & Est. & Est. & Prog. $^{1}$ & Est. \\
\hline \multicolumn{5}{|c}{ (Percentage change) } \\
Real GDP & 3.0 & 2.4 & 1.5 & 1.8 \\
CPI: end of period (EOP) & 29.7 & 39.1 & 15.0 & 12.8 \\
Nominal effective exchange rate (EOP) & -38.5 & -30.4 & $\ldots$ & 33.3 \\
\hline Sources: Guinean authorities and IMF staff estimates. & & & \\
1 Country Report No. 08/33. & & & & \\
\hline
\end{tabular}

39 percent,

falling below program projections. After stalling during social unrest early in 2007, economic activity made a timid recovery.

\section{Nevertheless, Guinea's external position is still precarious and two}

quantitative PCs were missed. Net international reserves (NIR) did not build up as expected. The central bank authorities were reluctant to intervene in a narrow market, because of the risk of liquidity injections and a run on the currency. Moreover, delays in payments of mining revenues and a higher-than-expected proportion of government spending in foreign currency at the end of 2007 led to the recurrence of external arrears to some multilateral creditors. These arrears were settled by end-March 2008. 


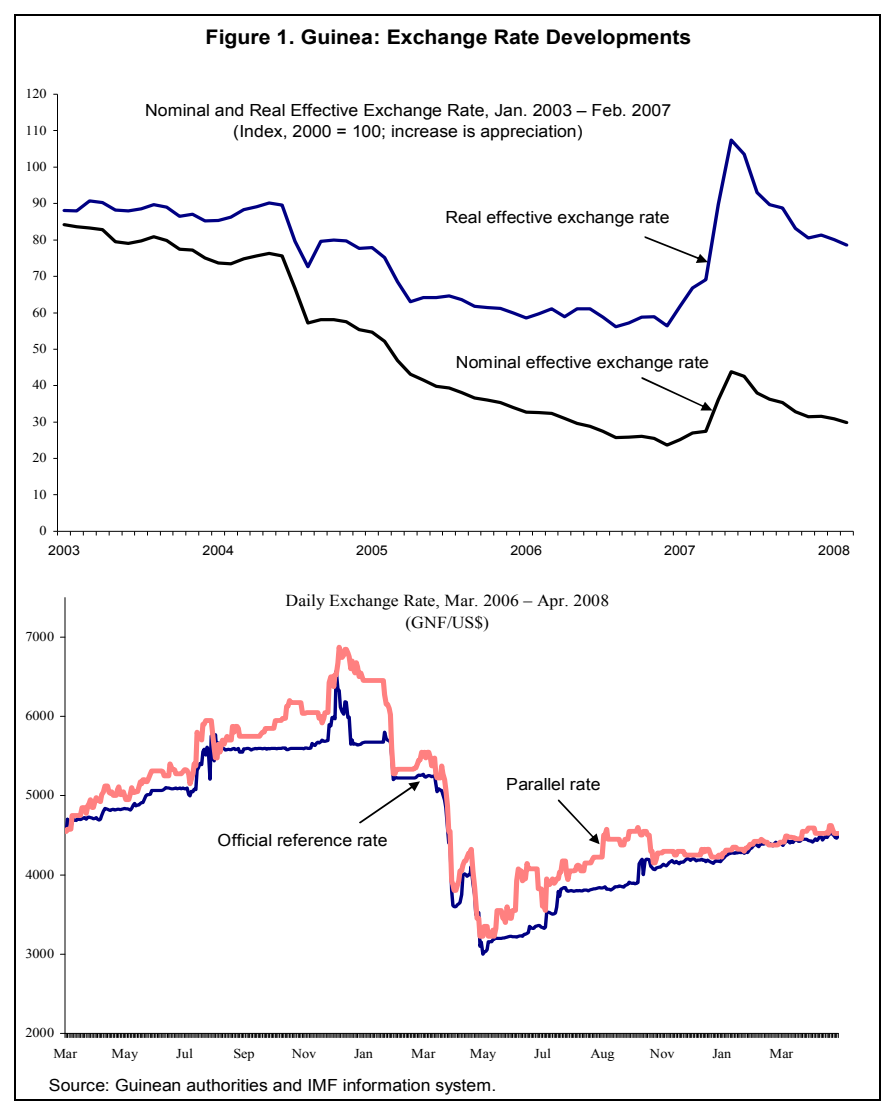

\section{The fiscal targets for $\mathbf{2 0 0 7}$ were broadly achieved, in spite of a revenue}

shortfall. The basic primary

fiscal balance improved by

2 percentage points of GDP,

exceeding the target by

0.2 percent of GDP. Currency

appreciation lowered the

taxable base (imports and

mining activity for the major

part) in Guinean francs,

bringing the government

revenue-to-GDP ratio below

target by 0.5 percentage points

(Text Table 2). Mining

revenues fell short also because

of technical interruptions in

production and cost overruns,

but intensified audits and a

buoyant telecommunication

\begin{tabular}{|lrrrr|}
\hline \multicolumn{5}{|c|}{ Text Table 2. Fiscal Performance, 2005-07 } \\
\hline & 2005 & 2006 & 2007 \\
\cline { 5 - 5 } & Est. & Est. & Prog. ${ }^{1}$ & Est. \\
\hline \multicolumn{5}{|c}{ (Percent of revised GDP) } \\
Revenue & 14.5 & 14.8 & 14.8 & 14.3 \\
Mining sector & 3.6 & 4.6 & 3.9 & 3.3 \\
Nonmining sector, of which: & 11.0 & 10.2 & 10.9 & 11.0 \\
Direct taxes & 1.7 & 1.9 & 2.1 & 2.5 \\
International trade & 3.0 & 3.0 & 3.0 & 2.7 \\
Total expenditures, of which: & 16.9 & 19.6 & 16.8 & 14.8 \\
Primary current expenditures & 9.1 & 11.3 & 9.9 & 8.9 \\
Priority sector spending & 3.9 & 4.0 & 4.3 & 4.3 \\
Basic primary fiscal balance & 3.5 & 1.5 & 3.5 & 3.7 \\
Domestic financing, of which: & 1.3 & 4.0 & 0.6 & -0.5 \\
Central bank & -0.2 & 6.2 & -0.4 & -1.1 \\
Commercial banks & 1.5 & -2.0 & 0.6 & 0.4 \\
External financing & 0.4 & -0.8 & -0.5 & 0.3 \\
\hline Sources: Guinean authorities and IMF staff estimates. & \\
1 Country Report No. 08/33. & & & & \\
\hline
\end{tabular}


sector boosted domestic taxation. The authorities cut nonpriority current spending to maintain budget discipline. Fiscal revenues in the first quarter of 2008 fell short of projections because of the lower taxable base and evaporating petroleum taxation revenues. The basic primary balance fell short of the end-March indicative target in spite of expenditure cuts

\section{Tightened internal controls and the zero government financing policy helped} the central bank bring monetary expansion under control. Growth in reserve money and broad money were kept below nominal GDP growth in 2007, reflecting the marginal increase in domestic credit and the low accumulation of foreign reserves. The credit market was affected by business uncertainties from social unrest earlier in the year, and by stepped-up enforcement of the capital-adequacy ratio by the central bank (BCRG). ${ }^{1}$ Commercial banks largely exceeded reserve requirements, and interest rates stabilized somewhat above inflation. In the first quarter of 2008, banks continued to accumulate foreign-currency denominated deposits with the central bank, which brought reserve and broad money above the end-March 2008 indicative targets, while their credit to the public and private sectors remained below program projections.

\section{Progress of structural reforms is broadly satisfactory and all structural PCs} and benchmarks for end-December 2007 were met (LOI, Table 2). An external audit conducted on end-December 2007 central bank data concluded that data provided to the Fund are consistent with the BCRG balance sheet (LOI, $\uparrow 4$ ). However, measures required to meet several structural benchmarks scheduled for early 2008 have been delayed.

\footnotetext{
${ }^{1}$ Compliance with prudential regulations on single-borrower limits or foreign exchange positions is limited, mainly because of lumpy external trade credit operations. Commercial banks' compliance with other prudential regulations improved in 2007
} 


\section{THE 2008 PROGRAM}

\section{A. Macroeconomic Framework}

7. Adverse developments in international markets have worsened economic prospects in 2008 and are putting pressure on the balance of payments (Text Table 3). The dramatic increase in food and fuel import prices is projected to increase the external current account deficit by about US\$190 million (4.3 percent of GDP), ${ }^{2}$ push up end-year CPI

\begin{tabular}{|c|c|c|c|c|c|}
\hline & \multirow{2}{*}{$\begin{array}{r}2007 \\
\text { Est. }\end{array}$} & \multicolumn{2}{|c|}{2008} & \multirow{2}{*}{$\begin{array}{l}2009 \\
\text { Proj. }\end{array}$} & \multirow{2}{*}{$\begin{array}{r}2010 \\
\text { Proj. }\end{array}$} \\
\hline & & Prog. & Proj. & & \\
\hline & \multicolumn{5}{|c|}{ (Percentage change) } \\
\hline Real GDP & 1.8 & 4.9 & 4.5 & 4.7 & 5.0 \\
\hline $\mathrm{CPI}$ (end of period) & 12.8 & 10.0 & 15.0 & 10.0 & 7.0 \\
\hline \multirow[t]{2}{*}{ Reserve money } & 11.0 & 14.5 & 22.2 & $\ldots$ & $\ldots$ \\
\hline & \multicolumn{5}{|c|}{ (Percent of revised GDP, unless otherwise indicated) } \\
\hline Government revenues & 14.3 & 16.2 & 15.6 & 16.5 & 16.9 \\
\hline Government expenditures & 14.8 & 19.4 & 18.4 & 18.6 & 18.9 \\
\hline Primary current expenditures & 8.9 & 10.5 & 10.4 & 10.6 & 10.6 \\
\hline Capital expenditures & 3.5 & 6.3 & 5.5 & 6.0 & 6.5 \\
\hline Basic primary fiscal balance & 3.7 & 3.7 & 3.5 & 4.0 & 4.2 \\
\hline Current account (incl. official transfers) & -2.0 & $-14.6^{1}$ & -5.4 & -6.8 & -6.3 \\
\hline Gross official reserves (months of imports) & 0.4 & 1.0 & 1.0 & 2.0 & 3.0 \\
\hline \multicolumn{6}{|c|}{$\begin{array}{l}\text { Source: Guinean authorities and IMF staff estimates and projections. } \\
{ }^{1} \text { Because of full revision of the balance of payments and national accounts, the program number } \\
\text { is not comparable with the estimates and projections. }\end{array}$} \\
\hline
\end{tabular}

inflation by 5 percentage points, and increase poverty incidence. ${ }^{3}$ Rising world fuel prices are gradually eroding the tax margin on the fixed pump price and fiscal revenue on top of the impact of the currency appreciation on the revenue base which carries through in 2008. Economic recovery may suffer from terms of trade losses and from the wait-and-see attitude of large foreign investors, especially in the mining sector.

\section{B. Fiscal Policy}

\section{By raising domestic petroleum prices by 63 percent on April $1^{\text {st }}$, 2008, the} government restored temporarily the normal petroleum taxation that was suspended in early 2007. Since then, however, world prices of oil have continued to increase, and the authorities decided to postpone the automatic adjustment of pump prices to endNovember 2008 (missing the end-June 2008 PC), stressing the risks tied to further domestic price increases in the present unstable social context. The impact of this postponement on government revenue in 2008 is estimated at about 0.3 percent of GDP.

\footnotetext{
${ }^{2}$ Based on the WEO prices from June 2008.

${ }^{3}$ According to World Bank simulations, poverty incidence could rise by 4 percentage points in the likely event the price of rice increases by half in 2008. Rice imports make almost up to 70 percent of all food imports.
} 
9. The authorities are making efforts to broadly maintain the fiscal targets.

The target for the basic primary balance has been lowered by 0.2 percentage points of GDP to 3.5 percent of GDP to make room for a policy response to the food and fuel price crisis (see below); the additional financing needs will be covered by external grants. The revenue shortfall will be offset in part by new tax administration measures and by cuts in capital and current expenditures, including mission travel (LOI, $₫ 10$ ). The fiscal program again refrains from new central bank financing of the budget. ${ }^{4}$

\begin{tabular}{|lrrr|}
\hline \multicolumn{4}{|c|}{ Text Table 4. Fiscal Performance, 2007-08 } \\
\hline & 2007 & 2008 \\
\cline { 2 - 4 } & Est. & Prog. $^{1}$ & Proj. \\
\hline & (Percent of revised & GDP) \\
Revenue & 14.3 & 16.2 & 15.6 \\
Mining sector & 3.3 & 4.1 & 3.7 \\
Nonmining sector & 11.0 & 12.1 & 11.9 \\
Total expenditures, of which: & 14.8 & 19.4 & 18.4 \\
Primary current expenditures & 8.9 & 10.5 & 10.4 \\
Basic primary fiscal balance & 3.7 & 3.7 & 3.5 \\
Overall balance, commitments basis & 0.3 & -1.4 & -1.0 \\
Domestic financing, of which: & -0.5 & -0.8 & -1.1 \\
Central bank & -1.1 & -0.5 & -0.5 \\
Commercial banks & 0.4 & 0.9 & 0.9 \\
External financing & 0.3 & -5.4 & -0.1 \\
Identified possible financing & $\ldots$ & 6.8 & 2.2 \\
Residual financing gap & $\ldots$ & 0.7 & 0.0 \\
\hline Sources: Guinean authorities and IMF staff estimates. & \\
1 Country Report No. 08/33. & & & \\
\hline
\end{tabular}

10. The authorities have further reinforced tax administration. Starting in May 2008, an international pre-inspection company, VERITAS, will conduct independent estimates of the value of imports. Moreover, the authorities expect to recover revenues from actions against the fraudulent transactions revealed by recent audits of ministerial departments.

\section{The authorities are finalizing with the World Bank and other donors a set of} measures to cushion the impact of higher food and petroleum prices. The bulk of the measures already adopted in the budget (for 0.4 percentage points of GDP) is aimed at civil servants. Customs duties on rice imports have also been reduced for a limited period. Other budget measures include: increased fellowships for university students, reduced transport prices for school students, "work for food" schemes. To address vulnerability to external food prices and supply constraints, it has also requested donor support to boost the supply response of agricultural production. Moreover, the government has already received a strong international response to its call for emergency food assistance to targeted vulnerable groups before the new crop season begins in September.

\section{The authorities have agreed to contain fiscal exposure to the public electricity} company (EDG). EDG's financial position has deteriorated because additional costs of oil inputs and increased access have not been matched by improvements in technical

\footnotetext{
${ }^{4}$ The authorities also plan to pay throughout 2008 the wage arrears claims made by the military (about 0.4 percent of GDP). These will be financed by additional resources from updated external debt relief projections, and would not affect the above-the-line fiscal targets.
} 
performance and commercial recovery rates. Short-term liquidity constraints had become so severe that BCRG granted EDG an emergency credit line in early 2008. The authorities agreed to freeze this credit line and adopt an emergency plan for EDG (a prior action for this review) that includes measures to reduce the rate of technical losses, improve billing and collection rates, combat fraud, and increase tariffs. They will also intensify the monitoring of EDG through regular financial reports (LOI, $\uparrow 14$ ).

\section{Monetary and Exchange Rate Policies}

\section{The monetary program seeks to limit year-end inflation to 15 percent (LOI, $\llbracket 6$} and 11) using indirect instruments of monetary policy. Reserve money growth, the anchor for monetary policy, will be aligned with nominal GDP growth. Authorities and staff concur that this will demand active liquidity management, including through improved forecasts, appropriate issuance of central bank bills, and close coordination with the Treasury. The BCRG is prepared to increase the flexibility of its auction mechanism in view of increasing the return of central bank paper (TRM) purchased by banks.

\section{The authorities intend to keep a flexible exchange rate regime while gradually} building up Guinea's external position to increase its resilience to exogenous shocks. The exchange rate has depreciated by about 8 percent since the beginning of 2008. In line with initial program objectives, the BCRG intends to gradually bring official reserves to one month of import cover by year-end. However, given the shortfalls in reserve accumulation through December 2007 and the increased balance of payments needs because of external shocks in the first half of 2008, the authorities have revised the reserve accumulation path throughout 2008 , and started to increase steadily foreign exchange purchases by the central bank. ${ }^{5}$ Other remedial actions including strengthened planning of, and monitoring and reporting on debt service payments and reserve accumulation will reduce the risk of program slippage. The mission encouraged the authorities to sterilize these interventions to keep the reserve money target within reach.

\section{Structural Reforms}

15. Central bank reforms are expected to continue at a fair pace. The authorities have adopted (i) standards for managing foreign reserves, as the safeguards assessment report recommended, and (ii) regulatory orders to improve transparency in the determination of the exchange rate. ${ }^{6}$ In conjunction with the ongoing review of foreign

\footnotetext{
${ }^{5}$ NIRs have increased by US\$24 million between end-December 2007 and end-May 2008, putting the end-June 2008 NIR target within reach.

${ }^{6}$ These measures reduce but do not eliminate the multiple currency practice arising from the risk of a deviation of the exchange rate used by the central bank in its foreign exchange transactions from the exchange rate used by commercial banks in transactions with their customers.
} 
exchange regulations, the BCRG drafted new instructions to pave the way for the development of an interbank foreign exchange market (e.g., financial market agreement). Moreover, it reorganized the foreign exchange department and created a foreign exchange commission that will oversee interbank market operations and ensure they accord with regulations set in the financial market agreement. Preparation of a new central bank law is underway with Fund technical assistance; the authorities intend to submit the draft to Parliament by end-November 2008 (thus missing the end-June 2008 PC).

16. Public finance management (PFM) is improving. A multiyear strategy to guide critically-needed PFM reform and achieve transparency and good governance of government operations will be adopted by end-June after consultation with donors. Its adoption is a prior action to conclude the first review. The authorities plan to adopt the new budget classification and definition of poverty-reducing expenditures in time for the preparation of the 2009 budget. Most ministries have been audited, and the authorities are moving to follow up on the recommendations.

17. The authorities are committed to speeding up mining sector reforms. The authorities now envisage adoption by the Council of Ministers of a standard mining contract based on international practices for end-July, and a revised mining code by end2008 (LOI, ๆ15). A joint World Bank/IMF mission is scheduled to provide technical assistance on a few outstanding issues, such as transfer pricing and sliding royalty scales. Guinea has agreed to be a pilot country for the World Bank-sponsored enhancing of the Extractive Industry Transparency Initiative (EITI), and to speed up the implementation and posting of EITI audits.

\section{E. External Policies and Outlook}

18. Guinea allowed the decree banning agricultural exports to lapse. The decree that prohibited exports of agricultural, forestry, and fishery products in early 2007 expired at year-end and was not renewed. As staff has advised, the authorities have resisted calls to re-impose an export ban on certain agricultural products such as rice.

\section{Guinea has benefited marginally from the worldwide boom in its commodity} export prices. Recent increases in world prices for aluminum and gold have largely escaped the country because of (i) increasing transportation and transformation costs; 
(ii) poorly-indexed country income shares in current mining contracts; and (iii) tax exemptions and transfer price practices.

20. Guinea is seeking additional financial assistance to address the negative shortterm impact of price shocks on the balance of payments. Additional debt relief and budget support pledged since the beginning of the year are expected to close the financing gap in 2008. Guinea received better-than-expected terms under the January 2008 Paris Club agreement that resumes bilateral debt relief in the context of the new PRGF arrangement, and the authorities have requested similar treatment for obligations to nonParis Club members. ${ }^{7}$ Several multilateral donors, including the AfDB, have increased interim HIPC relief on an exceptional basis (LOI, $\uparrow 18$ ). Development partners, including IDA and the EU are preparing additional balance of payments support operations that would cover the residual financial financing gap in 2008 in response to the food and fuel price crisis (Table 5). ${ }^{8}$ The authorities have also requested an augmentation of access to PRGF resources of 20 percent of quota in 2008, which would finance about a fifth of the balance of payment impact.

\section{Program Monitoring And Data}

21. The revised program incorporates proposed modifications to the end-June PCs for NIR and net domestic assets (NDA) of the central bank, and the basic fiscal primary balance (LOI, Table 1). The modification of the PC on NIR reflects the upward revision of oil and food prices that will limit BCRG capacity to accumulate foreign reserves. The PC on NDA and the target on reserve money are modified to reflect updated monetary projections. PCs for end-December 2008 are proposed for the fourth disbursement. The revised program includes also a new benchmark on central bank credit to public enterprises to curtail EDG's financial losses and their impact on the economy.

\section{The central bank action plan to improve internal controls, financial}

governance, and accountability is on track. Financial governance and internal accounting and control procedures were strengthened following recommendations of the safeguards assessment update and external audits. An internal audit charter was adopted and an audit committee created under the direct oversight of BCRG's Board to address the systemic override of controls and procedures unveiled by the safeguards assessment

\footnotetext{
${ }^{7}$ Guinea concluded debt agreements with three non-Paris Club creditors in 2006 and 2007. Those with Romania and China appear to be in line with HIPC terms. Regarding the agreement signed with Libya in November 2007, the authorities intend to seek further debt relief once Guinea reaches the HIPC completion point.

${ }^{8}$ Projections of emergency food aid in response to a government call for a US\$28 million assistance under the World Food Program are not yet reflected in the balance of payment projections for 2008.
} 
update. Accounting records have also been reconstructed to reflect the findings of the external audit of the 2006 accounts. It is expected that the audit of the financial statements for 2007 will confirm enhanced transparency in central bank accounts.

\section{The authorities are working with staff to improve the quality of statistics for} program monitoring. Substantial shortcomings in the assessment of trade flows, private transfers, income payments, and foreign direct investments (FDI) make balance of payments projections highly unreliable. The authorities should draft plans and mobilize human resources to implement the recommendations of the recent Fund technical assistance in this regard. Progress should continue in the area of national accounts (LOI, \19). ${ }^{9}$

\section{Staff Appraisal}

\section{Guinea's macroeconomic performance in the second half of 2007 was} satisfactory. Strict budget discipline and control of BCRG operations led to spectacular results on inflation while the economy was recovering. However, the December 2007 PCs on NIR and external arrears were missed, mainly because of unforeseen circumstances.

\section{Since January 2008 the authorities have weathered large and unexpected}

external shocks. Faced with surges in international fuel and food prices, the authorities reacted courageously by substantially increasing petroleum prices. Through close dialogue with trade unions and civil society, they resisted the temptation to reestablish an export ban on food products, while containing pressures for unaffordable mitigating measures.

Although the bulk of these measures targets the civil service, the authorities have also initiated measures to protect the poor and requested donors assistance to increase agricultural supply.

26. The authorities' commitment to further stabilize the economy is welcome. Continuation of strict fiscal and monetary policies is essential to meet inflation and international reserves targets, and should help FDI-led growth and poverty reduction to materialize.

27. The focus on public finance management is also commendable. The multiyear action plan for PFM is a blueprint to achieve measurable progress in budget preparation, execution, and monitoring. Better budget oversight, especially through tracking of poverty reduction expenditures, will also make it easier to mobilize budget support and debt relief.

\footnotetext{
${ }^{9}$ Following the revisions to the national accounts, nominal GDP has been revised downward, on average by about 8 percent in 2003-05.
} 
28. Reforms to improve the management of Guinea's abundant natural resources should be a high priority. The government should adopt a standard mining contract and revise mining taxation before new negotiations (and envisaged renegotiations of current contracts) take place. Adherence to the EITI will also make Guinea's business environment more transparent and spread the benefits of the country's natural resources.

29. Timely implementation of the central bank law and other reforms to improve central bank governance are critical. Several recommendations of the external auditor and the 2007 safeguard assessment update have already been implemented, notably the reinforcement of accounting, internal control, and internal audit functions. Another milestone will be the government's presentation to Parliament by November 2008 of a new law that ensures central bank independence and limits government financing.

30. The main risks to the program are a further worsening of the external environment, and political and social instability. The recent riots illustrate how Guinea's fragile environment can threaten economic stability and undermine progress toward reaching the HIPC completion point. In this context, staff supports the modifications to the program but stresses the need for the adoption of the petroleum price adjustment formula by November 2008 to avoid fiscal slippage.

31. Based on performance to date and remedial actions (including the clearance of external arrears, the recent build-up of NIRs, and actions being taken to meet the two structural PCs on the new test dates) and reiterated commitment to program implementation by the government, staff supports the authorities' requests for waivers for the nonobservance of two quantitative PCs and recommends completion of the first review under the PRGF arrangement.

32. In view of the adverse external shock that Guinea is experiencing, staff also supports the authorities' proposed modifications in the quantitative PCs for the third disbursement and their request for augmentation of access to PRGF resources. 
Table 1. Guinea: Selected Economic and Financial Indicators, 2005-10

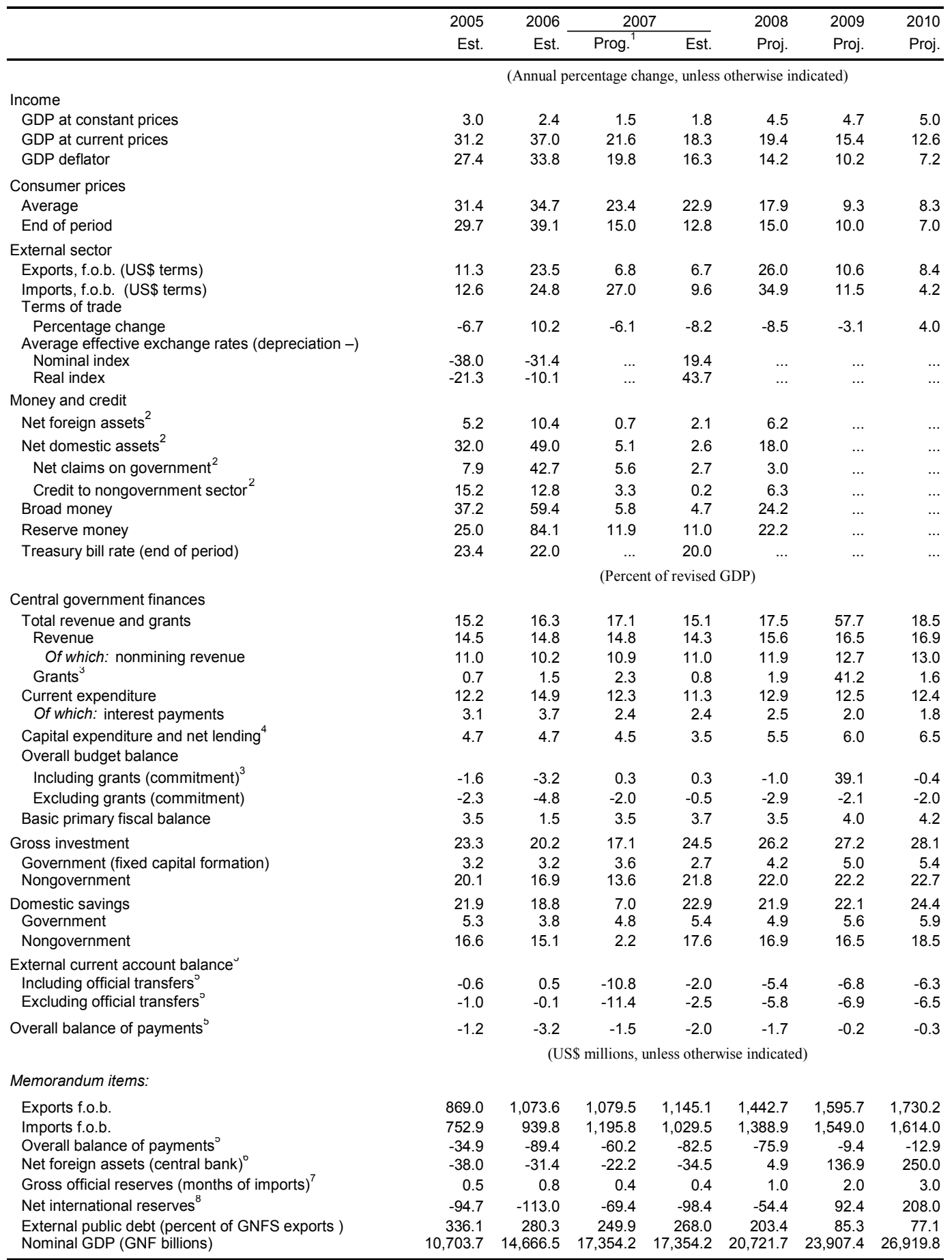

Sources: Guinean authorities and IMF staff estimates and projections.

${ }^{1}$ Country Report No. 08/33.

${ }^{2}$ Percent of broad money stock at the beginning of the period.

${ }^{3}$ Assumes that Guinea reaches the HIPC completion point at end-Dec. 2008 and the debt relief transactions will be booked in early 2009. Relief is shown as a stock of debt reduction.

${ }^{4}$ Includes expenditure for restructuring.

${ }^{5}$ Estimates and projections adjusted for profit repatriation by the mining companies, re-exports, and worker remittances.

${ }^{6}$ Gross foreign assets of the central bank less its foreign liabilities.

${ }^{7}$ Excluding imports for large mining projects and those financed by project loans from donors.

${ }^{8}$ Reserve assets of the central bank (i.e., external assets immediately available to and controlled by it) less its foreign and domestic liabilites in foreign exchange (incl. foreign exchange deposits of local banks). 
Table 2. Guinea: Financial Operations of the Central Government, 2005-10 (GNF billions)

\begin{tabular}{|c|c|c|c|c|c|c|c|c|c|c|c|}
\hline & \multirow{2}{*}{$\begin{array}{r}2005 \\
\text { Dec. } \\
\text { Est. }\end{array}$} & \multirow{2}{*}{$\begin{array}{r}2006 \\
\text { Dec. } \\
\text { Est. }\end{array}$} & \multicolumn{2}{|c|}{2007} & \multicolumn{5}{|c|}{$2008^{1}$} & \multirow{2}{*}{$\begin{array}{l}2009 \\
\\
\text { Dec. } \\
\text { Proj. }\end{array}$} & \multirow{2}{*}{$\begin{array}{l}2010 \\
\text { Dec. } \\
\text { Proj. }\end{array}$} \\
\hline & & & $\begin{array}{r}\text { Dec. } \\
\text { Prog. }^{2}\end{array}$ & $\begin{array}{r}\text { Dec. } \\
\text { Est. }\end{array}$ & $\begin{array}{r}\text { Mar. } \\
\text { Est. }\end{array}$ & $\begin{array}{l}\text { Jun. } \\
\text { Proj. }\end{array}$ & $\begin{array}{l}\text { Sep. } \\
\text { Proj. }\end{array}$ & $\begin{array}{l}\text { Dec. } \\
\text { Prog. }\end{array}$ & $\begin{array}{l}\text { Dec. } \\
\text { Proj. }\end{array}$ & & \\
\hline Revenue and grants & 1,631 & 2,398 & 2,967 & 2,620 & 663 & 1,690 & 2,584 & 3,748 & 3,620 & 13,787 & 4,973 \\
\hline Revenue & 1,557 & 2,172 & 2,569 & 2,477 & 650 & 1,516 & 2,304 & 3,362 & 3,230 & 3,940 & 4,552 \\
\hline Mining sector & 381 & 675 & 674 & 572 & 139 & 315 & 495 & 848 & 758 & 905 & 1,050 \\
\hline Nonmining sector & 1,176 & 1,497 & 1,895 & 1,905 & 510 & 1,201 & 1,809 & 2,514 & 2,472 & 3,036 & 3,502 \\
\hline Direct taxes & 181 & 280 & 368 & 427 & 108 & 253 & 358 & 444 & 469 & 541 & 609 \\
\hline Indirect taxes & 872 & 1,057 & 1,397 & 1,348 & 381 & 842 & 1,277 & 1,864 & 1,760 & 2,233 & 2,598 \\
\hline Goods and services & 554 & 620 & 879 & 875 & 268 & 525 & 784 & 1,150 & 1,079 & 1,379 & 1,577 \\
\hline International trade & 318 & 436 & 519 & 473 & 113 & 317 & 493 & 714 & 681 & 854 & 1,022 \\
\hline Nontax revenue & 123 & 161 & 130 & 130 & 21 & 106 & 174 & 206 & 243 & 262 & 295 \\
\hline Grants & 75 & 225 & 397 & 143 & 14 & 174 & 280 & 386 & 390 & 9,847 & 421 \\
\hline Project & 28 & 129 & 291 & 52 & 14 & 124 & 208 & 321 & 297 & 342 & 386 \\
\hline Program & 4 & 10 & 34 & 33 & 0 & 0 & 17 & 25 & 25 & 30 & 36 \\
\hline HIPC multilateral & 44 & 86 & 72 & 58 & 0 & 50 & 55 & 40 & 69 & 0 & 0 \\
\hline HIPC/MDRI completion point assistance ${ }^{3}$ & & & & & & & & & & 9,474 & \\
\hline Total expenditures and net lending & 1,808 & 2,871 & 2,914 & 2,566 & 685 & 1,774 & 2,742 & 4,030 & 3,820 & 4,440 & 5,088 \\
\hline Current expenditures & 1,304 & 2,188 & 2,139 & 1,954 & 480 & 1,214 & 1,902 & 2,724 & 2,676 & 2,998 & 3,342 \\
\hline Primary current expenditures & 975 & 1,652 & 1,718 & 1,541 & 416 & 992 & 1,560 & 2,183 & 2,156 & 2,526 & 2,853 \\
\hline Salary and wage & 333 & 444 & 643 & 607 & 146 & 391 & 620 & 869 & 881 & 1,017 & 1,145 \\
\hline $\begin{array}{l}\text { Goods and services } \\
\text { Of which: foreign-financed election spending }\end{array}$ & 396 & 810 & $\begin{array}{r}665 \\
58\end{array}$ & 559 & 198 & 424 & 651 & 888 & 872 & 1,020 & 1,155 \\
\hline Transfers and subsidies & 247 & 397 & 410 & 375 & 72 & 177 & 289 & 426 & 402 & 489 & 554 \\
\hline Interest on debt & 329 & 536 & 420 & 413 & 64 & 222 & 343 & 541 & 521 & 472 & 489 \\
\hline Domestic debt & 161 & 263 & 203 & 193 & 20 & 119 & 194 & 301 & 304 & 313 & 319 \\
\hline External debt & 168 & 274 & 218 & 220 & 44 & 104 & 148 & 240 & 217 & 159 & 169 \\
\hline Capital expenditures & 500 & 672 & 769 & 608 & 204 & 556 & 834 & 1,298 & 1,136 & 1,433 & 1,736 \\
\hline Domestically financed & 199 & 295 & 297 & 294 & 101 & 210 & 253 & 401 & 334 & 449 & 556 \\
\hline Externally financed & 301 & 377 & 472 & 314 & 103 & 346 & 581 & 897 & 802 & 984 & 1,180 \\
\hline Net lending & 3 & 11 & 6 & 5 & 0 & 4 & 6 & 8 & 8 & 9 & 10 \\
\hline Basic primary fiscal balance ${ }^{4}$ & 379 & 214 & 605 & 638 & 133 & 310 & 485 & 771 & 732 & 956 & 1,132 \\
\hline \multicolumn{12}{|l|}{ Overall balance, commitment basis } \\
\hline Excluding grants & -251 & -699 & -345 & -89 & -35 & -258 & -438 & -668 & -591 & -500 & -536 \\
\hline Including grants & -176 & -474 & 52 & 54 & -21 & -84 & -158 & -281 & -200 & 9,347 & -115 \\
\hline Financing & 176 & 474 & 12 & -38 & 34 & -23 & -36 & $-1,272$ & -255 & $-9,541$ & -35 \\
\hline Domestic financing & 134 & 586 & 106 & -82 & 41 & -9 & -64 & -159 & -234 & -357 & -535 \\
\hline Bank financing & 135 & 617 & 32 & -125 & 149 & 120 & 118 & 101 & 101 & -144 & -144 \\
\hline Central bank & -21 & 916 & -68 & -187 & 33 & 0 & -47 & -95 & -94 & -94 & -94 \\
\hline Of which: HIPC account & -25 & 74 & 0 & 0 & 0 & 0 & 0 & -5 & 0 & 0 & 0 \\
\hline Commercial banks & 156 & -300 & 100 & 62 & 116 & 120 & 165 & 196 & 195 & -50 & -50 \\
\hline Nonbank financing & -1 & -31 & 75 & 43 & -108 & -129 & -182 & -260 & -335 & -213 & -392 \\
\hline Privatization revenue & 88 & 285 & 177 & 189 & 3 & 30 & 30 & 125 & 81 & 3 & 3 \\
\hline Amortization of domestic debt & -83 & -241 & -240 & -237 & -60 & -146 & -219 & -288 & -313 & -216 & -395 \\
\hline Change in arrears & 0 & 0 & 76 & 75 & 0 & -18 & -53 & -102 & -88 & 0 & 0 \\
\hline Change in float ${ }^{5}$ & -35 & -92 & 27 & 30 & -52 & 5 & 59 & 5 & -16 & 0 & 0 \\
\hline Other & 29 & 17 & 35 & -14 & 0 & 0 & 0 & 0 & 0 & 0 & 0 \\
\hline External financing & 42 & -112 & -94 & 44 & -6 & -14 & 28 & $-1,113$ & -21 & $-9,185$ & 501 \\
\hline Drawings & 273 & 248 & 239 & 262 & 90 & 221 & 372 & 576 & 505 & 641 & 794 \\
\hline Amortization due ${ }^{3}$ & -433 & -831 & -633 & -637 & -136 & -228 & -362 & -625 & -573 & $-9,922$ & -437 \\
\hline Debt rescheduling and HIPC assistance ${ }^{6}$ & 143 & 103 & 47 & 83 & 44 & 775 & 800 & 193 & 828 & 96 & 143 \\
\hline Change in arrears $(-=$ reduction $)$ & 58 & 368 & 253 & 336 & -4 & -782 & -782 & $-1,257$ & -782 & 0 & 0 \\
\hline Errors and omissions ${ }^{5}$ & $\ldots$ & $\ldots$ & -65 & -16 & -13 & 0 & 0 & 0 & 0 & 0 & 0 \\
\hline Financing gap & 0 & 0 & 0 & 0 & 0 & 107 & 194 & 1,553 & 456 & 195 & 150 \\
\hline Identified financing & & & & & & & & 1,414 & 456 & & \\
\hline Residual financing gap & & & & & & & & 139 & 0 & 195 & 150 \\
\hline \multicolumn{12}{|l|}{ Memorandum items: } \\
\hline Priority sector spending ${ }^{7}$ & 416 & 586 & 740 & 744 & 191 & 493 & 763 & 1,057 & 1,057 & 1,232 & 1,401 \\
\hline Nominal GDP & 10,704 & 14,666 & 19,852 & 17,354 & 20,722 & 20,722 & 20,722 & 20,722 & 20,722 & 23,907 & 26,920 \\
\hline
\end{tabular}

Sources: Guinean authorities and IMF staff estimations and projections.

${ }^{1}$ Cumulative.

${ }^{2}$ Country Report No. 08/33.

${ }^{3}$ Assumes that Guinea reaches the HIPC Completion Point at end-Dec. 2008 and the debt relief transactions will be booked in early 2009

Relief is shown as a stock of debt reduction.

${ }^{4}$ Revenue minus noninterest expenditure and foreign-financed expenditures.

${ }^{5}$ For 2005-06, errors and omissions are included in the change in float.

${ }^{6}$ Committed debt relief.

${ }^{7}$ See the Technical Memorandum of Understanding (TMU) for a definition. 
Table 3. Guinea: Financial Operations of the Central Government, 2005-10

(Percent of revised GDP, unless otherwise indicated)

\begin{tabular}{|c|c|c|c|c|c|c|c|c|c|c|c|}
\hline & \multirow{2}{*}{$\begin{array}{l}2005 \\
\text { Dec. } \\
\text { Real. }\end{array}$} & \multirow{2}{*}{$\begin{array}{r}2006 \\
\text { Dec. } \\
\text { Real. }\end{array}$} & \multicolumn{2}{|c|}{2007} & \multicolumn{5}{|c|}{$2008^{1}$} & \multirow{2}{*}{$\begin{array}{l}2009 \\
\text { Dec. } \\
\text { Proj. }\end{array}$} & \multirow{2}{*}{$\begin{array}{l}2010 \\
\text { Dec. } \\
\text { Proj. }\end{array}$} \\
\hline & & & $\begin{array}{l}\text { Dec. } \\
\text { Prog. }^{2}\end{array}$ & $\begin{array}{r}\text { Dec. } \\
\text { Est. }\end{array}$ & $\begin{array}{c}\text { Mar. } \\
\text { Est. }\end{array}$ & $\begin{array}{l}\text { Jun. } \\
\text { Proj. }\end{array}$ & $\begin{array}{l}\text { Sep. } \\
\text { Proj. }\end{array}$ & $\begin{array}{l}\text { Dec. } \\
\text { Prog. }{ }^{2}\end{array}$ & $\begin{array}{l}\text { Dec. } \\
\text { Proj. }\end{array}$ & & \\
\hline Revenue and grants & 15.2 & 16.3 & 17.1 & 15.1 & 3.2 & 8.2 & 12.5 & 18.1 & 17.5 & 57.7 & 18.5 \\
\hline Revenue & 14.5 & 14.8 & 14.8 & 14.3 & 3.1 & 7.3 & 11.1 & 16.2 & 15.6 & 16.5 & 16.9 \\
\hline Mining sector & 3.6 & 4.6 & 3.9 & 3.3 & 0.7 & 1.5 & 2.4 & 4.1 & 3.7 & 3.8 & 3.9 \\
\hline Nonmining sector & 11.0 & 10.2 & 10.9 & 11.0 & 2.5 & 5.8 & 8.7 & 12.1 & 11.9 & 12.7 & 13.0 \\
\hline Direct taxes & 1.7 & 1.9 & 2.1 & 2.5 & 0.5 & 1.2 & 1.7 & 2.1 & 2.3 & 2.3 & 2.3 \\
\hline Indirect taxes & 8.1 & 7.2 & 8.1 & 7.8 & 1.8 & 4.1 & 6.2 & 9.0 & 8.5 & 9.3 & 9.7 \\
\hline Goods and services & 5.2 & 4.2 & 5.1 & 5.0 & 1.3 & 2.5 & 3.8 & 5.6 & 5.2 & 5.8 & 5.9 \\
\hline International trade & 3.0 & 3.0 & 3.0 & 2.7 & 0.5 & 1.5 & 2.4 & 3.4 & 3.3 & 3.6 & 3.8 \\
\hline Nontax revenue & 1.2 & 1.1 & 0.8 & 0.7 & 0.1 & 0.5 & 0.8 & 1.0 & 1.2 & 1.1 & 1.1 \\
\hline Grants & 0.7 & 1.5 & 2.3 & 0.8 & 0.1 & 0.8 & 1.4 & 1.9 & 1.9 & 41.2 & 1.6 \\
\hline Project & 0.3 & 0.9 & 1.7 & 0.3 & 0.1 & 0.6 & 1.0 & 1.5 & 1.4 & 1.4 & 1.4 \\
\hline Program & 0.0 & 0.1 & 0.2 & 0.2 & 0.0 & 0.0 & 0.1 & 0.1 & 0.1 & 0.1 & 0.1 \\
\hline HIPC multilateral & 0.4 & 0.6 & 0.4 & 0.3 & 0.0 & 0.2 & 0.3 & 0.2 & 0.3 & 0.0 & 0.0 \\
\hline HIPC/MDRI completion point assistance ${ }^{3}$ & & & & & & & & & & 39.6 & \\
\hline Total expenditures and net lending & 16.9 & 19.6 & 16.8 & 14.8 & 3.3 & 8.6 & 13.2 & 19.4 & 18.4 & 18.6 & 18.9 \\
\hline Current expenditures & 12.2 & 14.9 & 12.3 & 11.3 & 2.3 & 5.9 & 9.2 & 13.1 & 12.9 & 12.5 & 12.4 \\
\hline Primary current expenditures & 9.1 & 11.3 & 9.9 & 8.9 & 2.0 & 4.8 & 7.5 & 10.5 & 10.4 & 10.6 & 10.6 \\
\hline Salary and wage & 3.1 & 3.0 & 3.7 & 3.5 & 0.7 & 1.9 & 3.0 & 4.2 & 4.3 & 4.3 & 4.3 \\
\hline Goods and services & 3.7 & 5.5 & 3.8 & 3.2 & 1.0 & 2.0 & 3.1 & 4.3 & 4.2 & 4.3 & 4.3 \\
\hline Of which: foreign-financed election spending & & & 0.3 & & $\ldots$ & & . & & & & \\
\hline Transfers and subsidies & 2.3 & 2.7 & 2.4 & 2.2 & 0.3 & 0.9 & 1.4 & 2.1 & 1.9 & 2.0 & 2.1 \\
\hline Interest on debt & 3.1 & 3.7 & 2.4 & 2.4 & 0.3 & 1.1 & 1.7 & 2.6 & 2.5 & 2.0 & 1.8 \\
\hline Domestic debt & 1.5 & 1.8 & 1.2 & 1.1 & 0.1 & 0.6 & 0.9 & 1.5 & 1.5 & 1.3 & 1.2 \\
\hline External debt & 1.6 & 1.9 & 1.3 & 1.3 & 0.2 & 0.5 & 0.7 & 1.2 & 1.0 & 0.7 & 0.6 \\
\hline Capital expenditures & 4.7 & 4.6 & 4.4 & 3.5 & 1.0 & 2.7 & 4.0 & 6.3 & 5.5 & 6.0 & 6.4 \\
\hline Domestically financed & 1.9 & 2.0 & 1.7 & 1.7 & 0.5 & 1.0 & 1.2 & 1.9 & 1.6 & 1.9 & 2.1 \\
\hline Externally financed & 2.8 & 2.6 & 2.7 & 1.8 & 0.5 & 1.7 & 2.8 & 4.3 & 3.9 & 4.1 & 4.4 \\
\hline Net lending & 0.0 & 0.1 & 0.0 & 0.0 & 0.0 & 0.0 & 0.0 & 0.0 & 0.0 & 0.0 & 0.0 \\
\hline Basic primary fiscal balance ${ }^{4}$ & 3.5 & 1.5 & 3.5 & 3.7 & 0.6 & 1.5 & 2.3 & 3.7 & 3.5 & 4.0 & 4.2 \\
\hline \multicolumn{12}{|l|}{ Overall balance, commitments basis } \\
\hline Excluding grants & -2.3 & -4.8 & -2.0 & -0.5 & -0.2 & -1.2 & -2.1 & -3.2 & -2.9 & -2.1 & -2.0 \\
\hline Including grants & -1.6 & -3.2 & 0.3 & 0.3 & -0.1 & -0.4 & -0.8 & -1.4 & -1.0 & 39.1 & -0.4 \\
\hline Financing & 1.6 & 3.2 & 0.1 & -0.2 & 0.2 & -0.1 & -0.2 & -6.1 & -1.2 & -39.9 & -0.1 \\
\hline Domestic financing & 1.3 & 4.0 & 0.6 & -0.5 & 0.2 & 0.0 & -0.3 & -0.8 & -1.1 & -1.5 & -2.0 \\
\hline Bank financing & 1.3 & 4.2 & 0.2 & -0.7 & 0.7 & 0.6 & 0.6 & 0.5 & 0.5 & -0.6 & -0.5 \\
\hline Central bank & -0.2 & 6.2 & -0.4 & -1.1 & 0.2 & 0.0 & -0.2 & -0.5 & -0.5 & -0.4 & -0.3 \\
\hline Of which: HIPC account & -0.2 & 0.5 & 0.0 & 0.0 & 0.0 & 0.0 & 0.0 & 0.0 & 0.0 & 0.0 & 0.0 \\
\hline Commercial banks & 1.5 & -2.0 & 0.6 & 0.4 & 0.6 & 0.6 & 0.8 & 0.9 & 0.9 & -0.2 & -0.2 \\
\hline Nonbank financing & 0.0 & -0.2 & 0.4 & 0.2 & -0.5 & -0.6 & -0.9 & -1.3 & -1.6 & -0.9 & -1.5 \\
\hline Privatization revenue & 0.8 & 1.9 & 1.0 & 1.1 & 0.0 & 0.1 & 0.1 & 0.6 & 0.4 & 0.0 & 0.0 \\
\hline Amortization of domestic debt & -0.8 & -1.6 & -1.4 & -1.4 & -0.3 & -0.7 & -1.1 & -1.4 & -1.5 & -0.9 & -1.5 \\
\hline Change in arrears & 0.0 & 0.0 & 0.4 & 0.4 & 0.0 & -0.1 & -0.3 & -0.5 & -0.4 & 0.0 & 0.0 \\
\hline Change in float ${ }^{5}$ & -0.3 & -0.6 & 0.2 & 0.2 & -0.3 & 0.0 & 0.3 & 0.0 & -0.1 & 0.0 & 0.0 \\
\hline Other & 29 & 17 & 35 & -14 & 0 & 0 & 0 & 0 & 0 & 0 & 0 \\
\hline External financing & 0.4 & -0.8 & -0.5 & 0.3 & 0.0 & -0.1 & 0.1 & -5.4 & -0.1 & -38.4 & 1.9 \\
\hline Drawings & 2.6 & 1.7 & 1.4 & 1.5 & 0.4 & 1.1 & 1.8 & 2.8 & 2.4 & 2.7 & 3.0 \\
\hline Amortization due ${ }^{3}$ & -4.0 & -5.7 & -3.6 & -3.7 & -0.7 & -1.1 & -1.7 & -3.0 & -2.8 & -41.5 & -1.6 \\
\hline Debt rescheduling and HIPC assistance ${ }^{6}$ & 1.3 & 0.7 & 0.3 & 0.5 & 0.2 & 3.7 & 3.9 & 0.9 & 4.0 & 0.4 & 0.5 \\
\hline Change in arrears $(-=$ reduction $)$ & 0.5 & 2.5 & 1.5 & 1.9 & 0.0 & -3.8 & -3.8 & -6.1 & -3.8 & 0.0 & 0.0 \\
\hline Errors and omissions ${ }^{5}$ & $\ldots$ & $\ldots$ & -0.4 & -0.1 & -0.1 & 0.0 & 0.0 & 0.0 & 0.0 & 0.0 & 0.0 \\
\hline Financing gap & 0.0 & 0.0 & 0.0 & 0.0 & 0.0 & 0.5 & 0.9 & 7.5 & 2.2 & 0.8 & 0.6 \\
\hline Identified financing & & & & & & & & 6.8 & 2.2 & & \\
\hline Residual financing gap & & & & & & & & 0.7 & 0.0 & 0.8 & 0.6 \\
\hline \multicolumn{12}{|l|}{ Memorandum items: } \\
\hline Priority sector spending ${ }^{7}$ & 3.9 & 4.0 & 4.3 & 4.3 & 0.9 & 2.4 & 3.7 & 5.1 & 5.1 & $\ldots$ & .. \\
\hline Nominal GDP (revised) & 10,704 & 14,666 & 17,354 & 17,354 & 20,722 & 20,722 & 20,722 & 20,722 & 20,722 & 23,907 & 26,920 \\
\hline
\end{tabular}

Sources: Guinean authorities and IMF staff estimations and projections.

${ }^{1}$ Cumulative.

${ }^{2}$ Country Report No. 08/33.

${ }^{3}$ Assumes that Guinea reaches the HIPC completion point at end-Dec. 2008 and the debt relief transactions will be booked in early 2009.

Relief is shown as a stock of debt reduction.

${ }^{4}$ Revenue minus noninterest expenditure and foreign-financed expenditures.

${ }^{5}$ For 2005-06, errors and omissions are included in the change in float.

${ }^{6}$ Committed debt relief.

${ }^{7}$ See the Technical Memorandum of Understanding (TMU) for a definition. 


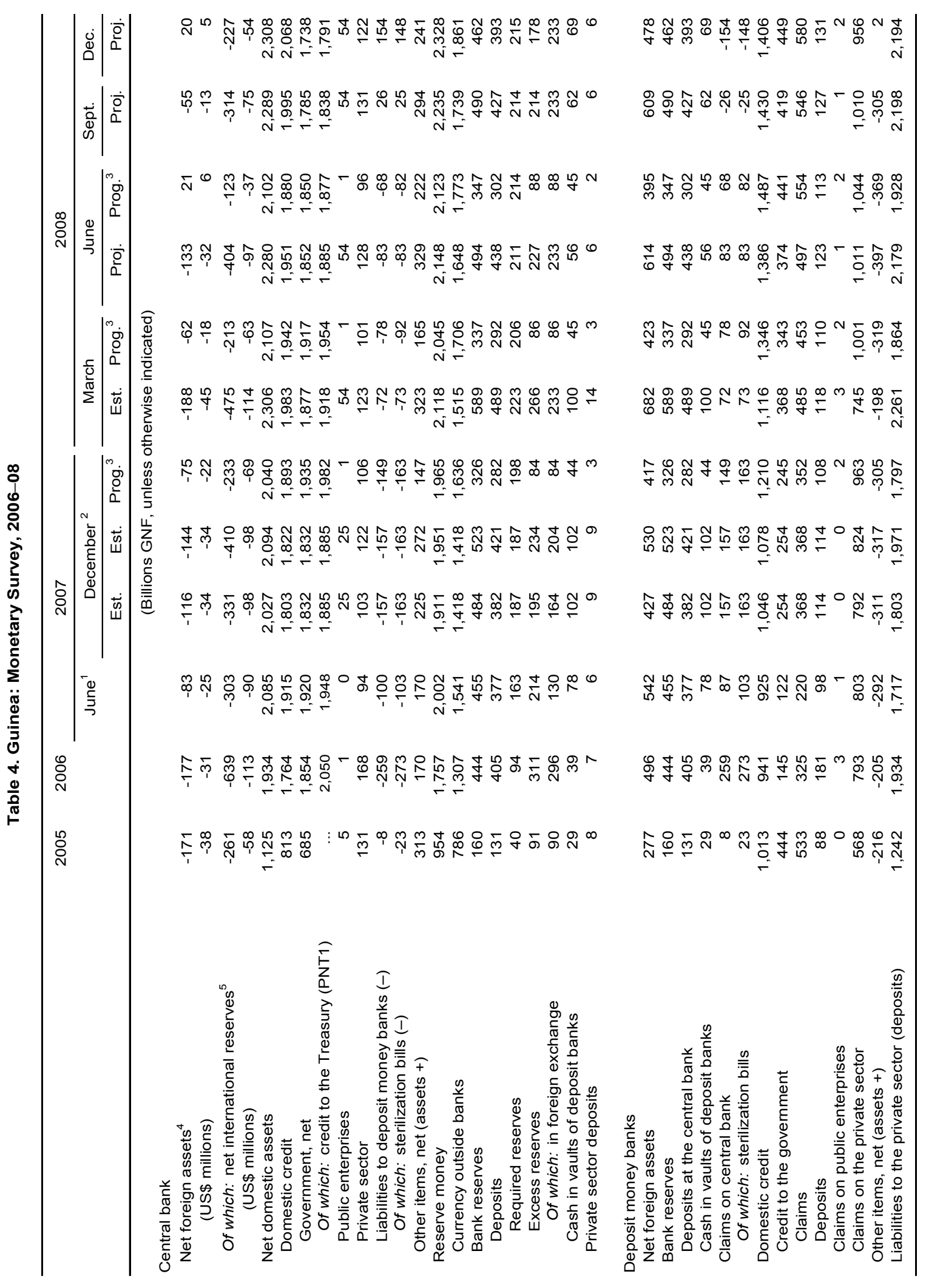




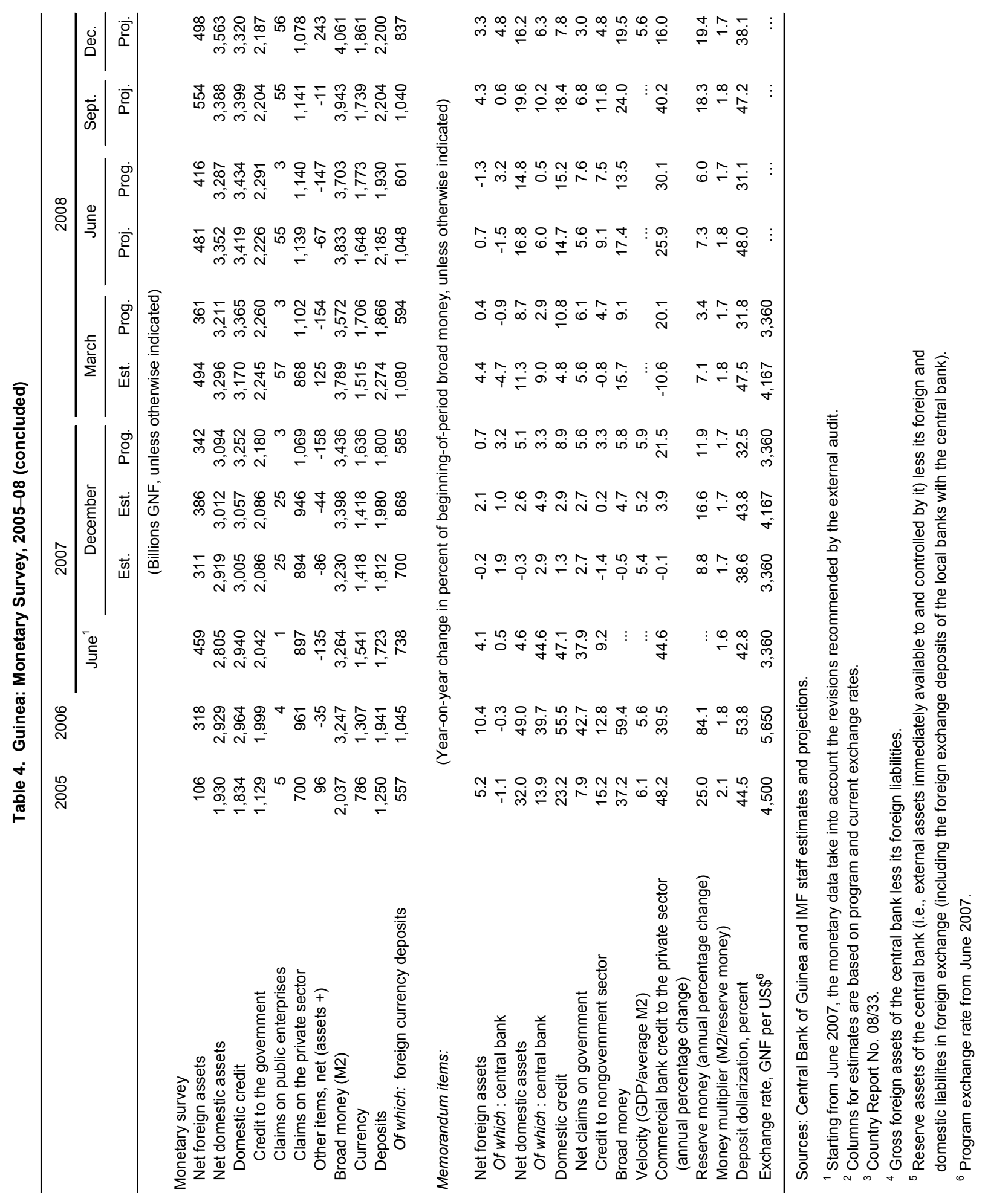


19

Table 5. Guinea: Balance of Payments, 2005-13 ${ }^{1}$

(US\$ millions, unless otherwise indicated)

\begin{tabular}{|c|c|c|c|c|c|c|c|c|c|}
\hline & $\begin{array}{r}2005 \\
\text { Est. }\end{array}$ & $\begin{array}{r}2006 \\
\text { Est. }\end{array}$ & $\begin{array}{r}2007 \\
\text { Est. }\end{array}$ & $\begin{array}{r}2008 \\
\text { Proj. }\end{array}$ & $\begin{array}{r}2009 \\
\text { Proj. }\end{array}$ & $\begin{array}{r}2010 \\
\text { Proj. }\end{array}$ & $\begin{array}{r}2011 \\
\text { Proj. }\end{array}$ & $\begin{array}{r}2012 \\
\text { Proj. }\end{array}$ & $\begin{array}{r}2013 \\
\text { Proj. }\end{array}$ \\
\hline Merchandise trade balance & 147 & 170 & 173 & 121 & 107 & 177 & 226 & 256 & 260 \\
\hline Exports, f.o.b & 869 & 1,074 & 1,145 & 1,443 & 1,596 & 1,730 & 1,920 & 2,116 & 2,303 \\
\hline Mining products & 782 & 963 & 1,067 & 1,358 & 1,496 & 1,615 & 1,807 & 1,987 & 2,166 \\
\hline Other & 87 & 111 & 78 & 85 & 99 & 115 & 113 & 129 & 136 \\
\hline Re-exports, f.o.b & 31 & 36 & 58 & 67 & 60 & 61 & 64 & 68 & 73 \\
\hline Imports, f.o.b. & -753 & -940 & $-1,030$ & $-1,389$ & $-1,549$ & $-1,614$ & $-1,758$ & $-1,928$ & $-2,115$ \\
\hline Food products & -126 & -137 & -142 & -194 & -205 & -216 & -222 & -230 & -237 \\
\hline Other consumption goods & -103 & -126 & -126 & -135 & -143 & -152 & -169 & -186 & -201 \\
\hline Petroleum goods & -156 & -198 & -258 & -445 & -500 & -479 & -507 & -539 & -578 \\
\hline Intermediate and capital goods & -367 & -479 & -504 & -614 & -700 & -767 & -861 & -974 & $-1,099$ \\
\hline Of which: imports for large mining projects & & -61 & -175 & -247 & -331 & -393 & -471 & -561 & -663 \\
\hline Services trade balance & -188 & -208 & -239 & -314 & -342 & -359 & -388 & -417 & -435 \\
\hline Services exports & 83 & 64 & 65 & 68 & 69 & 72 & 74 & 81 & 100 \\
\hline Services imports & -271 & -271 & -304 & -382 & -412 & -431 & -463 & -498 & -535 \\
\hline Of which: Imports for large mining projects & & -10 & -29 & -41 & -54 & -65 & -78 & -92 & -109 \\
\hline Income balance & -279 & -265 & -320 & -408 & -447 & -488 & -554 & -616 & -678 \\
\hline Of which: interest on public debt ${ }^{2}$ & -47 & -53 & -53 & -48 & -32 & -32 & -36 & -38 & -40 \\
\hline Transfers, Of which: & 303 & 318 & 302 & 363 & 369 & 356 & 370 & 386 & 402 \\
\hline Net private transfers & 290 & 299 & 280 & 343 & 363 & 350 & 363 & 378 & 392 \\
\hline Official transfers & 1 & 2 & 8 & 5 & 6 & 7 & 7 & 9 & 10 \\
\hline HIPC Initiative assistance (multilat.) & 12 & 17 & 14 & 15 & 0 & 0 & 0 & 0 & 0 \\
\hline \multicolumn{10}{|l|}{ Current account } \\
\hline Including official transfers & -17 & 15 & -84 & -238 & -314 & -314 & -346 & -390 & -451 \\
\hline Excluding imports for large mining projects & -17 & 85 & 121 & 50 & 71 & 144 & 203 & 263 & 320 \\
\hline Excluding official transfers & -30 & -4 & -105 & -258 & -320 & -320 & -354 & -399 & -461 \\
\hline Capital account & 8 & 25 & 12 & 64 & 1,932 & 71 & 77 & 84 & 92 \\
\hline Public transfers (project grants) & 8 & 25 & 12 & 64 & 66 & 71 & 77 & 84 & 92 \\
\hline HIPC completion point and MDRI debt relief ${ }^{3}$ & 0 & 0 & 0 & 0 & 1,866 & 0 & 0 & 0 & 0 \\
\hline Financial account & -94 & -98 & -25 & 99 & $-1,628$ & 230 & 265 & 313 & 382 \\
\hline Public (medium and long term) & -43 & -111 & -89 & -24 & $-1,795$ & 66 & 83 & 120 & 120 \\
\hline Project-related loans & 75 & 48 & 63 & 109 & 124 & 146 & 175 & 210 & 210 \\
\hline Program financing & 0 & 0 & 0 & 0 & 0 & 0 & 0 & 0 & 0 \\
\hline Amortization due ${ }^{2}$ & -118 & -159 & -152 & -133 & $-1,919$ & -80 & -92 & -90 & -90 \\
\hline Of which: HIPC completion point and MDRI & & & & & & & & & \\
\hline Public (short term) & 0 & 0 & 0 & 0 & 0 & 0 & 0 & 0 & 0 \\
\hline Direct and other private investment (net) & -81 & 26 & 29 & 94 & 128 & 122 & 135 & 143 & 209 \\
\hline Of which: large mining projects & 0 & 71 & 262 & 356 & 416 & 495 & 593 & 706 & 834 \\
\hline Private short term & 31 & -13 & 36 & 28 & 39 & 43 & 46 & 50 & 53 \\
\hline Errors and omissions & 69 & -31 & 13 & 0 & 0 & 0 & 0 & 0 & 0 \\
\hline Overall balance & -35 & -89 & -82 & -76 & -9 & -13 & -4 & 7 & 23 \\
\hline Financing & 35 & 89 & 82 & -72 & -134 & -108 & -14 & -9 & -25 \\
\hline Change in net foreign assets, Of which: & -23 & -7 & -7 & -92 & -153 & -134 & -31 & -26 & -41 \\
\hline Fund repayments $^{3}$ & -26 & -19 & -21 & -18 & -34 & 0 & 0 & 0 & 0 \\
\hline Change in gross official reserves & 5 & 3 & 6 & -76 & -119 & -134 & -31 & -26 & -37 \\
\hline Changes in arrears & 16 & 71 & 58 & -168 & 0 & 0 & 0 & 0 & 0 \\
\hline Debt rescheduling & 37 & 22 & 29 & 29 & 19 & 26 & 17 & 17 & 16 \\
\hline HIPC interim assistance & 5 & 4 & 2 & 159 & 0 & 0 & 0 & 0 & 0 \\
\hline Financing gap & 0 & 0 & 0 & 148 & 144 & 120 & 17 & 2 & 2 \\
\hline Identified financing, Of which: & & & & 148 & & & & & \\
\hline Additional debt relief BAD & & & & 8 & & & & & \\
\hline Paris Club Agreement & & & & 35 & & & & & \\
\hline Comparable treatment non- $\mathrm{PC}$ members & & & & 14 & & & & & \\
\hline Sectoral support IDA & & & & 20 & & & & & \\
\hline Support European Union & & & & 18 & & & & & \\
\hline New PRGF disbursements & & & & 53 & 21 & 21 & 0 & 0 & 0 \\
\hline Nonidentified external financing & 0 & 0 & 0 & 0 & 123 & 100 & 17 & 2 & 2 \\
\hline \multicolumn{10}{|l|}{ Memorandum items: } \\
\hline \multicolumn{10}{|l|}{ Current account-GDP ratio (percent) } \\
\hline Including official transfers & -0.6 & 0.5 & -2.0 & -5.4 & -6.8 & -6.3 & -6.4 & -6.6 & -7.0 \\
\hline Excluding imports for large mining projects & -0.6 & 3.0 & 2.9 & 1.1 & 1.5 & 2.9 & 3.8 & 4.5 & 5.0 \\
\hline Excluding official transfers & -1.0 & -0.1 & -2.5 & -5.8 & -6.9 & -6.5 & -6.6 & -6.8 & -7.2 \\
\hline Overall balance (percent of GDP) & -1.2 & -3.2 & -2.0 & -1.7 & -0.2 & -0.3 & -0.1 & 0.1 & 0.4 \\
\hline Exports-GDP ratio (percent) & 32.4 & 40.3 & 29.1 & 33.9 & 36.0 & 36.4 & 37.0 & 37.4 & 37.4 \\
\hline Imports-GDP ratio (percent) & -34.9 & -42.9 & -32.1 & -39.8 & -42.4 & -41.3 & -41.2 & -41.2 & -41.3 \\
\hline External medium- and long-term public debt & 3,199 & 3,188 & 3,242 & 3,072 & 1,420 & 1,389 & 1,330 & 1,277 & 1,184 \\
\hline Percent of GDP & 108.9 & 113.0 & 78.0 & 69.0 & 30.7 & 28.1 & 24.6 & 21.7 & 18.5 \\
\hline Debt-service ratio, before interim debt relief ${ }^{4}$ & 20.2 & 20.4 & 18.8 & 13.2 & 117.2 & 6.3 & 6.5 & 5.9 & 5.5 \\
\hline Gross reserves & 53.7 & 51.0 & 45.2 & 137.5 & 240.2 & 374.2 & 405.1 & 431.1 & 467.7 \\
\hline Months of imports of the following year ${ }^{5}$ & 0.5 & 0.8 & 0.4 & 1.0 & 2.0 & 3.0 & 3.1 & 3.1 & 3.1 \\
\hline Revised nominal GDP (US\$ millions) & 2,937 & 2,820 & 4,157 & 4,454 & 4,624 & 4,945 & 5,395 & 5,882 & 6,416 \\
\hline
\end{tabular}

Sources: Guinean authorities and IMF staff estimates and projections.

Figures adjusted for profit repatriation by the mining companies, re-exports, and worker remittances.

${ }^{2}$ Debt service payments on public and publicly guaranteed debt.

${ }^{3}$ Assumes that Guinea reaches the HIPC Completion Point at end-Dec. 2008 and the debt relief transactions will be booked in early 2009. Relief is shown as a stock of debt reduction.

${ }^{4}$ For 2009, includes the HIPC completion point and MDRI debt relief.

${ }^{5}$ Percent of exports of goods and nonfactor services, excluding imports for large mining projects and those financed by project loans from donors. 


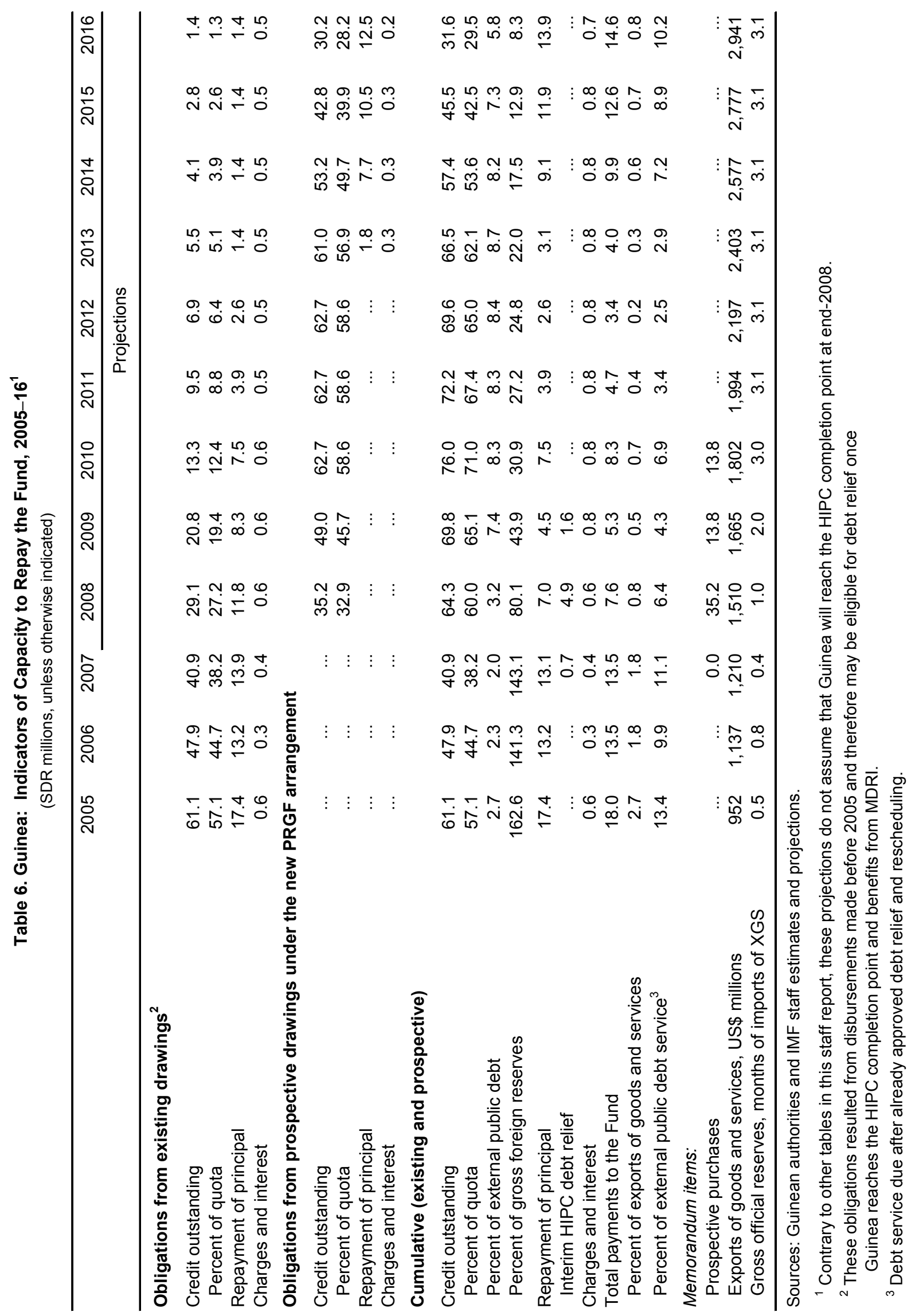


Table 7. Guinea: Millennium Development Goals, 1990-2015

\begin{tabular}{|c|c|c|c|c|c|c|c|}
\hline & 1990 & 1995 & 2000 & 2005 & $\begin{array}{c}2005 \\
\text { Sub- } \\
\text { Saharan } \\
\text { Africa } \\
\end{array}$ & $\begin{array}{c}2010 \\
\text { PRSP }\end{array}$ & $\begin{array}{c}2015 \\
\text { MDG } \\
\text { Target } \\
\end{array}$ \\
\hline Goal 1: Eradicate extreme poverty and hunger & & & & & & & halve \\
\hline Income share held by lowest $20 \%$ & .. &.. & .. & 7.0 &.. & $\ldots$ & \\
\hline Malnutrition prevalence, weight for age ( $\%$ of children under 5 ) & 26.8 &.. & 32.7 & .. & 29.6 & $\ldots$ & 13.4 \\
\hline Poverty gap at $\$ 1$ a day (PPP) (\%) & .. &.. & .. & .. & .. & $\ldots$ & $\ldots$ \\
\hline Poverty headcount ratio at $\$ 1$ a day (PPP) (\%) & .. &.. & .. & .. & 41.1 & $\ldots$ & $\ldots$ \\
\hline Poverty headcount ratio at national poverty line (\%) & .. & 40.0 & .. & .. & .. & $\ldots$ & $\ldots$ \\
\hline Prevalence of undernourishment (\%) & 39 & 31 & .. & 24 & 30 & $\ldots$ & 19.5 \\
\hline Incidence of poverty & & & 49.2 & 53.6 & & 49.7 & $\ldots$ \\
\hline Goal 2: Achieve universal primary education & & & & & & & 100 \\
\hline Literacy rate, youth total (\% of people ages $15-24)$ & 44 & .. & .. & 47 & 73 & & 100 \\
\hline Persistence to grade 5 , total ( $\%$ of cohort) & 59 &.. & .. & 76 & .. & & 100 \\
\hline Primary completion rate, total ( $\%$ of relevant age group) & 19 & 20 & 33 & 55 & 58 & 79 & 100 \\
\hline School enrollment, primary (\% net) & 27 &.. & 47 & 67 & 66 & 90 & 100 \\
\hline share of current expenditures on education in State equity resources & $\cdots$ & $\cdots$ & $\cdots$ & 14.2 & $\cdots$ & 18.9 & $\cdots$ \\
\hline Goal 3: Promote gender equality and empower women & & & & & & & 100 \\
\hline Proportion of seats held by women in national parliament (\%) & .. & 7 & $19 / 114$ & $22 / 114$ & 16 & $50 / 114$ & 100 \\
\hline Ratio of girls to boys in primary and secondary education (\%) & 45 & .. & 63 & 74 & 86 & 92 & 100 \\
\hline Ratio of young literate females to males (\% ages $15-24)$ & 43 &.. & .. & 57 & 88 & & \\
\hline $\begin{array}{l}\text { Share of women employed in the nonagricultural sector (\% of total } \\
\text { nonagricultural employment) }\end{array}$ & 30.3 &.$\cdot$ & .. & .. & .. & & \\
\hline Goal 4: Reduce child mortality & & & & & & & $>75 \%$ reduction \\
\hline Immunization, measles ( $\%$ of children ages $12-23$ months) & 35 & 61 & 42 & 71 & 64 & 80 & \\
\hline Mortality rate, infant (per 1,000 live births) & 139 & 124 & 110 & 91 & 96 & 70 & \\
\hline Mortality rate, under 5 (per 1,000$)$ & 234 & 209 & 183 & 163 & 163 & 130 & 78 \\
\hline \multicolumn{8}{|l|}{ Goal 5: Improve maternal health } \\
\hline Births attended by skilled health staff ( $\%$ of total) & 31 & .. & 35 & 38 & 45 & 40 & \\
\hline Maternal mortality ratio (modeled estimate, per 100,000 live births) & .. &.. & 740 & 980 & .. & 700 & $>75 \%$ reduction \\
\hline Goal 6: Combat HIVIAIDS, malaria, and other diseases & & & & & & & halt/reverse \\
\hline Contraceptive prevalence (\% of women ages $15-49$ ) & 2 &.. & 6 & 7 & 23 & & \\
\hline Incidence of tuberculosis (per 100,000 people) & 130 & 158 & 193 & 236 & 348 & & halt/reverse \\
\hline Prevalence of HIV, female (\% ages $15-24)$ &.. &.. & .. & 1.4 & .. & & halt/reverse \\
\hline Prevalence of HIV, pregnant womenl (\% of population ages $15-49)$ & .. & .. & 2.8 & .. & $\ldots$ & $<2.8 \%$ & halt/reverse \\
\hline Tuberculosis cases directly observed (\%) &.. & 43 & 54 & 56 & 49 & & halt/reverse \\
\hline \multicolumn{8}{|l|}{ Goal 7: Ensure environmental sustainability } \\
\hline CO2 emissions (metric tons per capita) & 0.2 & 0.2 & 0.2 & 0.1 & 0.8 & & \\
\hline Forest area ( $\%$ of land area) & 30 &.. & 28 & 27 & 27 & & \\
\hline GDP per unit of energy use (constant 2000 PPP \$ per kg of oil equivalent) & .. & .. & .. & .. & 2.8 & & \\
\hline Improved sanitation facilities (\% of population with access) & 14 &.. & .. & 18 & 37 & & 57 \\
\hline Improved water source (\% of population with access) & 44 &.. & .. & 50 & 56 & & 72 \\
\hline Nationally protected areas ( $\%$ of total land area) & .. &.. & .. & 6.4 & 11.3 & & \\
\hline Proportion of population with access to a beter sanitation system (urban/rural) &.. &.. & .. & 70.1 & $\ldots$ & & \\
\hline \multicolumn{8}{|l|}{ Goal 8: Develop a global partnership for development } \\
\hline Aid per capita (current US\$) & 47 & 55 & 18 & 19 & 44 & & to increase \\
\hline Debt service (PPG and IMF only, \% of exports, excl. workers' remittances) & 19.6 & 24.3 & 14.2 & 11.3 & 7.4 & 10 & \\
\hline Fixed line and mobile phone subscribers (per 1,000 people) & 2 & 2 & 8 & 20 & 142 & & \\
\hline Internet users (per 1,000 people) & 0 & 0 & 1 & 5 & 29 & & \\
\hline Personal computers (per 1,000 people) & .. & 1 & 3 & 5 & 15 & & \\
\hline Total debt service ( $\%$ of exports of goods, services, and income) & 20.0 & 25.0 & 20.4 & 19.9 & 8.8 & & \\
\hline Unemployment, youth female (\% of female labor force, ages 15-24) &.. &.. & .. &.. & .. & & \\
\hline Unemployment, youth male (\% of male labor force ages $15-24)$ & .. &.. & .. & .. & .. & & \\
\hline Unemployment, youth total (\% of total labor force ages $15-24)$ &.$\cdot$ &.. & .. &.$\cdot$ &. & & \\
\hline \multicolumn{8}{|l|}{ Other goals and indicators } \\
\hline Fertility rate, total (births per woman) & 6.5 & 6.3 & 5.6 & 5.6 & 5.3 & & \\
\hline GNI per capita, Atlas method (current US\$) & 430 & 490 & 400 & 420 & 746 & & \\
\hline GNI, Atlas method (current US\$ billions) & 2.6 & 3.7 & 3.4 & 3.9 & 554.4 & & \\
\hline Gross capital formation ( $\%$ of GDP) & 17.5 & 16.6 & 22.0 & 12.0 & 19.4 & & \\
\hline Life expectancy at birth, total (years) & 47 & 51 & 53 & 54 & 47 & & \\
\hline Literacy rate, adult total ( $\%$ of people ages 15 and above) & 27 &.. & .. & 29 & 61 & 50 & \\
\hline Population, total (millions) & 6.2 & 7.5 & 8.4 & 9.4 & 743.1 & & \\
\hline Trade (\% of GDP) & 61.5 & 45.3 & 52.3 & 55.7 & 67.3 & & \\
\hline
\end{tabular}

Sources: World Development Indicators database, April 2007; and Guinean authorities, Second Poverty Reduction Strategy Paper, August 2007.

${ }^{1}$ Figures in italics refer to periods other than those specified. 
Table 8. Guinea: Disbursements Under the PRGF Arrangement, 2007-10 (SDR millions)

\begin{tabular}{|c|c|c|}
\hline Date & Disbursement & Subject to \\
\hline December 21, 2007 & 6.885 & Approval of arrangement \\
\hline July 28, 2008 & 17.595 & $\begin{array}{l}\text { First review and performance criteria (PCs) } \\
\text { for end-December } 2007\end{array}$ \\
\hline December 2008 & 17.595 & Second review and PCs for end-June 2008 \\
\hline May 2009 & 6.885 & Third review and PCs for end-December 2008 \\
\hline November 2009 & 6.885 & Fourth review and PCs for end-June 2009 \\
\hline May 2010 & 6.885 & Fifth review and PCs for end-December 2009 \\
\hline November 2010 & 6.885 & Sixth review and PCs for end-June 2010 \\
\hline Total disbursement ${ }^{1}$ & 69.615 & \\
\hline
\end{tabular}

Source: IMF.

${ }^{1}$ The total disbursement is equivalent to 65 percent of Guinea's quota. 


\section{APPENDIX I. LETTER OF INTENT}

Conakry, June 27, 2008.

Mr. Dominique Strauss-Kahn

Managing Director

International Monetary Fund

Washington, D.C., 20431, USA

Dear Sir,

1. Further to our letter of December 4, 2007, and the accompanying Memorandum on Economic and Financial Policies (MEFP), we are hereby reporting the progress made in implementing the three-year program supported by the International Monetary Fund under the Poverty Reduction and Growth Facility (PRGF). Implementation of our new Poverty Reduction Strategy that underpins this program and achievement of the completion point under the HIPC Initiative are our government's major priorities. The Prime Minister appointed on May 20 has confirmed the determination of the newly formed consensus government to implement this program, keep up the good performance achieved, and continue the implementation of reforms we started with the support of our foreign partners.

2. Macroeconomic performance in the fourth quarter of 2007 broadly met program targets. Mining sector activity strengthened, which led to a slight increase in the expected GDP growth rate of 1.5 percent in 2007. Inflation declined below the target level (the yearon-year increase in prices was less than 13 percent in December compared with 15 percent envisaged in the program) as a result of implementation of economic stabilization policies and the good performance of the national currency. The budget deficit and growth of the money supply were kept within program limits. However, the external position remains very tenuous.

3. Most of the quantitative performance criteria established for end-December 2007 were met (Table 1). Thus, despite the government's lower-than-expected mining revenue, we maintained the basic fiscal balance above the program floor as a result of good year-end expenditure control. Similarly, the Treasury's budgetary float remained largely below the ceiling we had set. Unfortunately, two criteria were not met. The target for accumulation of net international reserves was not met due to higher-than-expected public expenditure in foreign exchange at year-end. Furthermore, it was difficult to monitor this objective because of a misunderstanding on the definition of this criterion in the program. Inadequate foreign exchange reserves led to the accumulation of external payment arrears to certain multilateral creditors from December 31, 2007. However, the arrears were paid in full on March 31, 2008. 
4. The structural reform agenda is moving ahead and the structural performance criteria were met (Table 2). The prohibition of exports of agricultural products from forestry and fishing was lifted on December 31, 2007. Also, the audit firm confirmed the consistency of the data used to calculate the quantitative performance criteria at end-2007 with the central bank's balance sheet. The preliminary audit report of the central bank's financial statements at end-December 2007 will be transmitted to the staff by end-June 2008. The two structural benchmarks for the first quarter of 2008 were met with a slight delay. The upgrading to international standards of surveillance, management and monitoring of international reserve accounts was completed with the creation of an investment committee on May 12, 2008. In order to restore the government's cash unit, the BCRG instructed the commercial banks on March 13, 2008 to close all the accounts of nonautonomous public entities and to transfer the balances to the treasury account.

5. The government has updated the macroeconomic framework taking into account, in particular, the impact of the unexpected increase in world oil and food prices on our already fragile economy. In 2008 these increases will have a strong negative impact on the balance of payments and will reduce our growth by a half-point of GDP to 4.5 percent. Mostly as a result of imported inflation, the year-on-year increase in prices should reach 15 percent at year-end instead of the 10 percent initially projected.

6. Despite this exogenous shock, we expect to limit the changes to our main economic policy objectives for 2008 in order to reestablish macroeconomic stability and strengthen our external position. The government's basic primary balance will be maintained at its 2007 level (a reduction of 0.2 percentage points of GDP compared with the original program target) in order to create enough fiscal space to accommodate accompanying measures. The growth of base money will be limited to that of nominal GDP, and the target for accumulation of foreign exchange reserves will be kept at one month of imports.

7. On the fiscal front, revenue projections were revised downward to take into account the impact of the improved performance of the Guinean franc in 2007 on our tax base, and the outturn of the first quarter of 2008. Increased efforts to collect mining revenue (especially through tax audits), and the expected effects of the implementation by the international company VERITAS of a program to audit imports in May, will partially offset these losses. The program to strengthen tax and customs administrations will be continued, controls on exemptions will be strengthened, and the recommendations of IMF technical assistance missions will be implemented as planned. We will organize quarterly monitoring of the execution of performance contracts approved by the two revenue collection departments at the beginning of the year.

8. We have introduced measures to cope with the surge in international prices for oil and food products. The freezing of petroleum products pump prices placed the budget at great risk. On April 1, 2008 normal taxes on all petroleum products have been restored causing a significant increase in pump prices. However, in the current social context it seems 
indispensable to postpone the implementation of the mechanism to adjust these prices on a monthly basis to reflect world prices to end-November (an end-June structural performance criterion). The revenue loss resulting from this delay could reach 0.3 percent of GDP, if international oil prices remain at the current level.

9. With the assistance of our development partners we will put in place an action plan to improve the targeting of accompanying measures to benefit the poor. Meanwhile, to cushion the impact of the increase in prices at the pump on transport users, we have increased the transport allowances for government employees and student scholarships and have granted subsidies for school transport. To mitigate the impact of the surge in prices of basic foodstuffs such as rice we have decided to suspend import duties on this product for 4 months (until the next harvest of rice in October). We also launched, with World Bank support, targeted aid programs in urban area school canteens and in prenatal health centers, as well as "food for work" programs. All in all, the costs of these measures will amount to about 0.4 percentage points of GDP ${ }^{1}$, of which about slightly less than half will be financed by the World Bank through budget support. In addition, we plan to mobilize emergency food aid and support to finance projects geared toward increasing agricultural production by making seeds, fertilizers, and pesticides more readily available.

10. To finance the remaining cost of the accompanying measures and take into account the downward revision of revenue in 2008, we decided to reduce expenditures by an amount equivalent to 0.5 percentage points of GDP. About one-fourth of those reductions will come from freezing a portion of budget allocations that had been earmarked for hiring new civil servants and creating incentive bonuses in the civil service. The government will continue to execute the budget without net monetary financing from the BCRG. Following the recent turmoil in the military over wage arrears dating back to 1996, and to restore social peace, we have recognized these arrears (about 0.4 percent of GDP) and agreed to gradually reimburse them in 2008 , by using the additional resources from updated external debt relief projections.

11. The BCRG will continue to implement a prudent monetary policy that is consistent with the goal of reducing structural inflation. It will use all available instruments to keep the money supply within the limits of the original program for 2008 , through proactive management of bank liquidity, including, if necessary, by improving the attractiveness for banks of monetary regulation securities. Monetary and foreign exchange policies will be closely coordinated and will continue to be based on adherence to market mechanisms.

12. Macroeconomic policy in the first half of 2008 will be consistent with the performance criteria and quantitative benchmarks for end-June, as defined in the attached

\footnotetext{
${ }^{1}$ We are also mobilizing emergency food assistance targeted to the poorest from WFP (World Food Program) and other UN agencies of the United Nations.
} 
Table 1. These criteria have been revised to reflect adjustments of our policies described above. Moreover, the lag in late 2007, the size of the exogenous shock on international food and oil prices, and delays in mobilizing international aid to cope with this situation have led us to revise downward the end-June 2008 target of net international reserves accumulation. However, we have prepared a monthly "catch-up" plan for 2008 that helped us accumulate net international reserves since April 2008. Between end-December 2007 and end-May 2008 we have accumulated US\$24 million.

13. Improved fiscal management remains a priority for government action. The multiyear plan that we prepared with the assistance of our main development partners will be adopted by the Council of Ministers by end-June, 2008 (cf. Table 3 on prior actions for the first review). Its execution and implementation of the IMF's technical assistance recommendations will be closely monitored.

14. The financial situation of the Guinean electricity company Électricité de Guinée (EDG) deteriorated in 2007. Work on rehabilitating the existing infrastructure made it possible to bring a few thermal and hydro units back into the network, increasing electricity production by about 21 percent. Although overall revenues have increased by 26 percent in 2007, and transmission and distribution losses were reduced, the progress made was not sufficient to improve EDG's financial situation in light of prevailing tariffs and the strong rise in the petroleum import price and the cost of spare parts. As a result, the increased production and higher fuel prices inflated operating deficits (by about 0.15 percent of GDP) and deepened EDG's overdraft at the BCRG. The continued increase in fuel prices and the practice of charging 80 percent of private subscribers (representing 30 percent of sales) a flat rate for electricity usage may worsen the situation in 2008. Short-term measures are needed to stem the financial hemorrhage, which threatens to compromise the progress made as well as our fiscal and external balances. Therefore, total outstanding debt of public enterprises (mostly EDG) owed to the BCRG has been frozen at its end-April level (new quantitative benchmark). Moreover, the Council of Ministers will approve an emergency plan by endJune 2008 (prior action) which includes measures to (i) significantly reduce the rate of technical losses; (ii) improve billing and collection rates, including by installing meters; (iii) combat fraud; and (iv) increase tariffs. For better monitoring of the financial situation, EDG will submit a management report to the government within one month from the end of each quarter, starting in July 2008 in a format that will be developed with assistance from the World Bank.

15. Continued efforts by the government to achieve good management and transparency in extractive industries are key to developing our resources and improving the business climate. The Council of Ministers will adopt by end-July a standard mining contract based on best international practices, which will serve as the basis for the ongoing renegotiation of current mining arrangements and negotiation of new contracts. To complement this approach, draft mining and oil codes will be completed by the end of the year with technical 
assistance from the World Bank and the IMF. Transparency of the revenue the government earns from mining activity was further enhanced with the publication of the audit report for 2005. The results of the 2006 audit will be finalized and published by end-November, 2008 .

16. Other actions to improve governance and transparency are ongoing. The audit of government bodies initiated by the government has been completed, except for the Ministry of Public Works, Urban Development and Housing. The irregularities uncovered by these audits are subject to collection actions. The government intends to speedily implement recommendations that the government's inspectorate general will finalize by August 2008 on the follow-up of these ministerial audits, in particular regarding administrative sanctions, collection of misappropriated government assets, and elimination of ghost workers. The actions we intend to take in this area should yield at least GF10 billion for the government in 2008.

17. The management and governance of the central bank are also being enhanced largely in compliance with the recommendations of the BCRG's external auditors and the IMF's safeguards assessment mission. BCRG staff have prepared a draft law establishing the central bank that guarantees BCRG independence and the transparency of its operations. After it is discussed with social partners, this draft will be adopted by the Council of Ministers and submitted to the National Assembly by end-November 2008 (a structural performance criterion for end-June 2008). The three-month slippage with respect to the structural performance criterion set in the original program makes it possible for Guinea to receive IMF technical assistance.

18. We will continue our efforts to consolidate the sustainability of our external debt. We will continue in particular to refrain from contracting or renewing any nonconcessional external loans to the government or to the central bank or guaranteed by them. We have entered into bilateral talks with our Paris Club creditors to finalize the January 2008 agreement (agreements have been already signed with four countries) and have asked our bilateral creditors that are not members of the Paris Club to provide us with debt restructuring deals on comparable terms in order to reach an equitable solution regarding the pending debt. We have obtained from the World Bank, the African Development Bank (AfDB) and the Arab Bank for Economic Development in Africa (BADEA) supplemental interim assistance under the HIPC Initiative, and we will continue to request from our multilateral creditors debt treatment that is aligned with the Initiative. The financing gap of the 2008 program will be covered through budgetary support from our main external partners, in particular the World Bank, the European Union, and France.

19. Because improvement in the coverage and quality of statistical data is crucial for program monitoring, our efforts in this area are continuing as planned. We have just started a major revision of the national accounts based on SNA 1993. Following the recommendations of a recent IMF mission on balance of payments statistics, we will develop an improvement plan and launch the necessary surveys (in particular of enterprises making direct investments 
in Guinea) to correct the main deficiencies and will produce a quarterly balance of payments data that meet the minimum standards of quality within three months after the end of September 2008. We will immediately start collecting the necessary data to document PRSP monitoring indicators and prepare the report on the first year of PRSP implementation.

20. In order to strengthen program monitoring and coordination at the highest level, the committee that has been created to monitor the program will provide us with a bi-weekly progress report on program implementation, including the payment of external debt obligations (to prevent accumulation of new arrears). A monthly meeting of the committee, which we will attend with the IMF representative in Conakry, will also be organized to examine the documentation prepared by our staff with respect to the requirements set forth in the Technical Memorandum of Understanding. Attached are the paragraphs of the TMU that were revised to fine tune the program adjustors and strengthen ours commitments regarding the reporting of data to monitor the program.

21. In light of the good overall execution of our program, and of the corrective actions we have taken to remedy the problems and delays in meeting the two quantitative performance criteria, we request the necessary waivers to conclude the first review. In response to the recent external shocks and to cover the residual financing gap, we are requesting an exceptional increase in access to PRGF resources of 20 percent of our quota, equivalent to SDR 21.42 million, in the form of an increment of SDR10.71 million in the two disbursements scheduled for 2008. The second and third reviews under the PRGF arrangement will be carried out no later than end-December 2008 and end-June 2009 respectively.

Sincerely yours,

/sgd/

Daouda Bangoura Governor of the BCRG
$/ \operatorname{sgd} /$

Ousmane Doré

Minister of Economy, Finance, and Planning

Attachment: Revisions to the Technical Memorandum of Understanding 


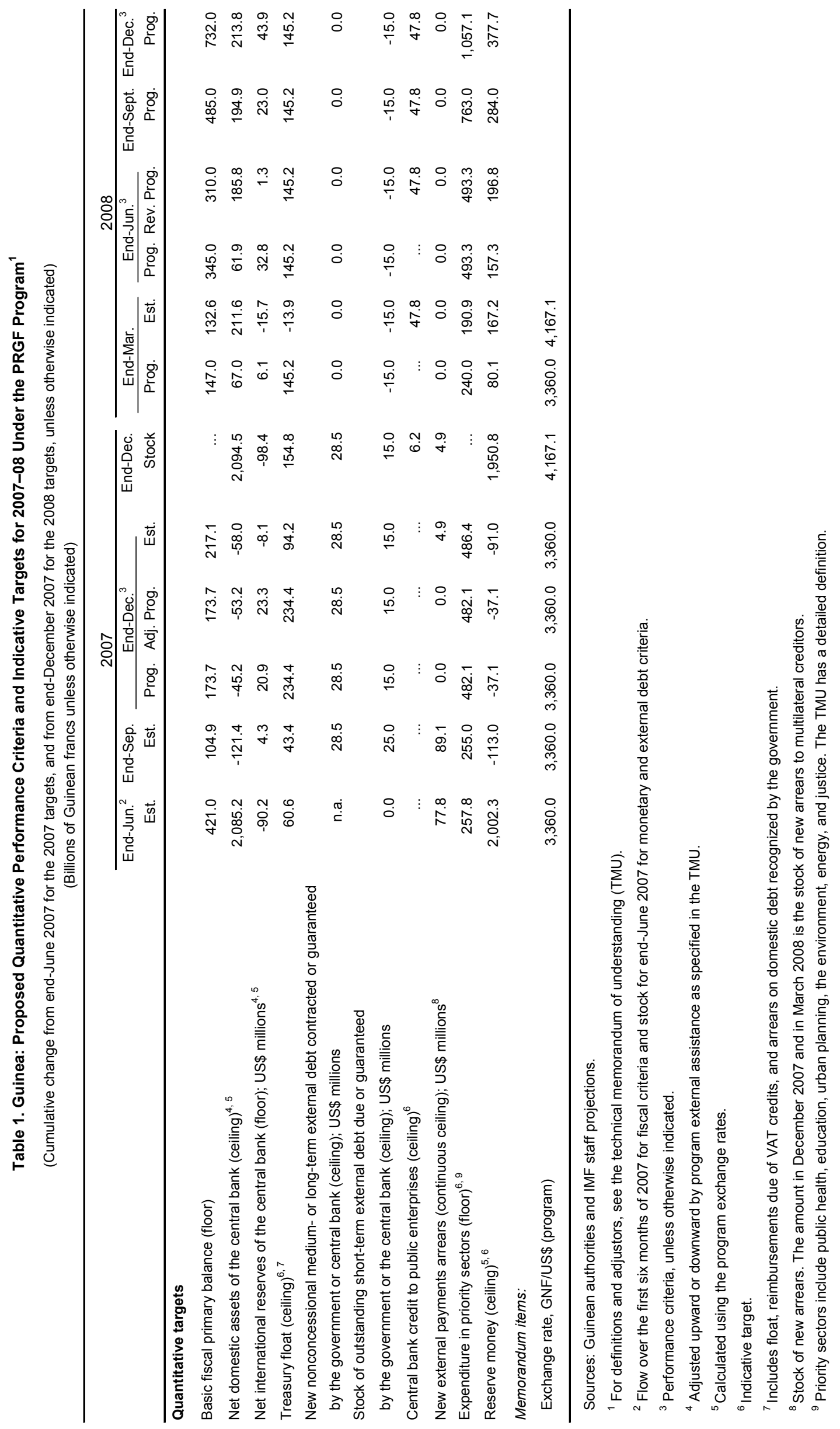




\section{Table 2. Guinea: Structural Performance Criteria and Benchmarks December 2007-December 2008}

\begin{tabular}{|c|c|c|}
\hline Action & $\begin{array}{l}\text { Performance } \\
\text { Criterion / } \\
\text { Benchmark }\end{array}$ & Status \\
\hline \multicolumn{3}{|l|}{ Continuous } \\
\hline $\begin{array}{l}\text { Deposit of PRGF disbursements in a separate account at the } \\
\text { Bank for International Settlements (BIS). Withdrawals from } \\
\text { this account will be subject to the express authorization of the } \\
\text { Governor or Vice Governor of the BCRG. }\end{array}$ & $\begin{array}{l}\text { Performance } \\
\text { Criterion }\end{array}$ & Met. \\
\hline $\begin{array}{l}\text { - No payment by the central bank for the account of the } \\
\text { government without the prior signature of the Minister of } \\
\text { Economic, Finance, and Planning or his official representative. }\end{array}$ & $\begin{array}{l}\text { Performance } \\
\text { Criterion }\end{array}$ & Met. \\
\hline - Ad hoc tax and customs exemptions are prohibited. & $\begin{array}{l}\text { Performance } \\
\text { Criterion }\end{array}$ & Met. \\
\hline - Extrabudgetary expenditure is prohibited. & $\begin{array}{l}\text { Performance } \\
\text { Criterion }\end{array}$ & Met. \\
\hline $\begin{array}{l}\text { - The BCRG will ensure the compliance of commercial banks } \\
\text { with the required reserves ratio on their deposits and the } \\
\text { system of sanctions provided for that purpose. }\end{array}$ & Benchmark & Met. \\
\hline \multicolumn{3}{|l|}{ December 31, 2007} \\
\hline $\begin{array}{l}\text { - Lift the prohibition against exports of agricultural, forestry, and } \\
\text { fishery products. }\end{array}$ & $\begin{array}{l}\text { Performance } \\
\text { Criterion }\end{array}$ & Met. \\
\hline $\begin{array}{l}\text { Issue a ministerial decree stating which expenditures are } \\
\text { eligible for simplified procedures and do not require prior } \\
\text { authorization. }\end{array}$ & Benchmark & Met. \\
\hline \multicolumn{3}{|l|}{ January 31, 2008} \\
\hline $\begin{array}{l}\text { Apply international standards to the oversight, management, } \\
\text { and accounting of the foreign exchange reserves in } \\
\text { accordance with the recommendations contained in the report } \\
\text { on safeguard measures. }\end{array}$ & Benchmark & $\begin{array}{l}\text { Met with delay. The investment } \\
\text { committee was created on } \\
\text { May } 12,2008 .\end{array}$ \\
\hline \multicolumn{3}{|l|}{ February 29, 2008} \\
\hline $\begin{array}{l}\text { Close all accounts of nonautonomous government entities at } \\
\text { commercial banks, except those explicitly required by donors. }\end{array}$ & Benchmark & $\begin{array}{l}\text { Met with delay. An instruction was sent } \\
\text { to commercial banks on } \\
\text { March } 13,2008 \text {. }\end{array}$ \\
\hline \multicolumn{3}{|l|}{ March 31, 2008} \\
\hline $\begin{array}{l}\text { - Independent verification by an external auditor of central bank } \\
\text { balance sheet data that will be used to calculate quantitative } \\
\text { performance criteria the (i.e., net international reserves and } \\
\text { net domestic assets) at end-December } 2007 \text {. }\end{array}$ & $\begin{array}{l}\text { Performance } \\
\text { Criterion }\end{array}$ & Met. \\
\hline \multicolumn{3}{|l|}{ April 30, 2008} \\
\hline $\begin{array}{l}\text { - Adopt in the Council of Ministers a standard mining contract } \\
\text { based on international practices. }\end{array}$ & Benchmark & $\begin{array}{l}\text { Not met. Expected to be adopted by } \\
\text { July } 31,2008 .\end{array}$ \\
\hline
\end{tabular}




\begin{tabular}{|c|c|c|}
\hline Action & $\begin{array}{l}\text { Performance } \\
\text { Criterion I } \\
\text { Benchmark }\end{array}$ & Status \\
\hline \multicolumn{3}{|l|}{ June 30, 2008} \\
\hline $\begin{array}{l}\text { - Submission of a draft law to Parliament amending the charter } \\
\text { of the central bank to strengthen its independence and limit } \\
\text { financing of the central government budget by the central } \\
\text { bank. }\end{array}$ & $\begin{array}{l}\text { Performance } \\
\text { Criterion }\end{array}$ & $\begin{array}{l}\text { Pending. A draft law will be submitted } \\
\text { to Parliament by November } 30,2008 \text {. }\end{array}$ \\
\hline $\begin{array}{l}\text { Reinstate the mechanism for the monthly determination of oil } \\
\text { prices based on a pricing formula. }\end{array}$ & $\begin{array}{l}\text { Performance } \\
\text { Criterion }\end{array}$ & $\begin{array}{l}\text { Pending. The mechanism will be } \\
\text { reinstated by end-November. }\end{array}$ \\
\hline $\begin{array}{l}\text { - Adopt a budget classification based on the IMF Government } \\
\text { Finance Statistics Manual } 2001 \text { (particularly the economic and } \\
\text { functional classifications) and define poverty-reducing } \\
\text { expenditure based on the functional classification. }\end{array}$ & Benchmark & \\
\hline $\begin{array}{l}\text { - Finalize and publish the results of the audit of mining sector } \\
\text { revenue and expenditure in } 2006 \text { in the context of the } \\
\text { Extractive Industries Transparency Initiative. }\end{array}$ & Benchmark & $\begin{array}{l}\text { Pending. Expected to be adopted by } \\
\text { November } 30,2008 .\end{array}$ \\
\hline \multicolumn{3}{|l|}{ August 31, 2008} \\
\hline $\begin{array}{l}\text { - Publish the central bank's audited financial statements for } \\
2006 \text { and } 2007 \text { on its website, together with the opinion of the } \\
\text { external auditor. }\end{array}$ & Benchmark & \\
\hline \multicolumn{3}{|l|}{ November 30, 2008} \\
\hline $\begin{array}{l}\text { - Submission of a draft law to Parliament amending the charter } \\
\text { of the central bank to strengthen its independence and limit } \\
\text { financing of the central government budget by the central } \\
\text { bank. }\end{array}$ & $\begin{array}{l}\text { Performance } \\
\text { Criterion }\end{array}$ & Reset from end-June \\
\hline $\begin{array}{l}\text { - Reinstate the mechanism for the monthly determination of oil } \\
\text { prices based on a pricing formula. }\end{array}$ & $\begin{array}{l}\text { Performance } \\
\text { Criterion }\end{array}$ & Reset from end-June \\
\hline \multicolumn{3}{|l|}{ December 31, 2008} \\
\hline $\begin{array}{l}\text { - Adopt an overall strategy and a rate policy for the electricity } \\
\text { sector. }\end{array}$ & Benchmark & \\
\hline
\end{tabular}


Table 3. Guinea: Status of Prior Actions for the First Review of the PRGF Arrangement

\begin{tabular}{|l|l|}
\hline Action & Status \\
\hline - Approve in the Council of Ministers an emergency plan which includes & \\
measures to (i) significantly reduce the rate of technical losses; \\
(ii) improve billing and collection rates, including by installing meters; \\
(iii) combat fraud; and (iv) increase tariffs. \\
- Adopt in the Council of Ministers the multiyear strategic plan for public \\
finance management. & \\
\end{tabular}




\section{Attachment I. Amendments to the Technical Memorandum of Understanding (TMU)}

June 27, 2008

1. Paragraphs 5, 8, 10, 15, and 16 and Table 1 of the Technical Memorandum of Understanding attached to our December 4, 2007 letter, and to the Memorandum of Economic and Financial Policies (MEFP) are modified by paragraphs 2, 3, 4, 5, and 6 and Table 1 as follows.

2. The net international reserves (NIR) of the BCRG are by definition, equal to the difference between the reserve assets of the BCRG (i.e., external assets immediately available to and controlled by the BCRG, in accordance with the fifth edition of the IMF Balance of Payments Statistics Manual), and the foreign exchange liabilities of the BCRG vis-à-vis residents and nonresidents (including the foreign exchange deposits of local banks with the BCRG, and BCRG's liabilities to the IMF). In the context of the program, BCRG reserve assets in gold will be valued at the price in effect on June 29, 2007 (US\$650.50 per ounce) for the second half of 2007 and at the price on December 31, 2007 (US\$833.75 per ounce) for 2008. On the dates of valuation, the equivalent in U.S. dollars of other reserve assets and liabilities in foreign exchange will be calculated at the program exchange rate, i.e. for the second half of 2007 at the exchange rate in effect on June 29, 2007 between the U.S. dollar and the Guinean franc (GF 3,360/US\$), the SDR (US\$1.51557/SDR), the euro (US\$1.3505/€), and the other currencies published in International Financial Statistics; and for 2008, at the exchange rate in effect on December 31, 2007 between the U.S. dollar and the Guinean franc (GF 4,167.1/US\$), the SDR (US\$1.5803/SDR), the euro (US\$1.4721/€), and the other currencies published in International Financial Statistics.

3. External payments arrears of the government and BCRG include all external debt service obligations falling due and not paid (principal and interest) on loans contracted or guaranteed by the government or BCRG, fines unpaid at their term, and interest charges stemming from these loans. For the purpose of this performance criterion, an obligation falling due and not paid after 30 days, and which is not subject to rescheduling negotiation will be considered arrears.

4. Payments pending are outstanding expenditures assumed by the Treasury (see definition below) and not yet paid, including reimbursements of VAT credits due, and arrears on domestic debt that are recognized by the government; wage bill outlays are excluded.

5. The NIR and NDA targets of the program are calculated on the basis of the projected amounts of net external assistance (see Table below). For purposes of the program, net external assistance is defined as the difference between (a) total budgetary assistance (grants and loans); and (b) total external debt service payments due after debt relief. 
Projected Amounts of Net External Assistance (Cumulative, US\$ millions)

\begin{tabular}{lrrrr}
\hline & Mar. & Jun. & Sept. & Dec. \\
\hline Net external assistance & -23.4 & -45.1 & -54.2 & -50.5 \\
Budgetary assistance (grants + loans) & 0.0 & 0.0 & 3.7 & 42.8 \\
Debt service payments & -23.4 & -45.1 & -57.9 & -93.4 \\
\hline
\end{tabular}

6. The floor on NIR and the ceiling on NDA will be subject to simultaneous adjustment if disbursements of net external assistance (as defined above) differ from program projections. The floor on NIR will be adjusted upward (downward) and the ceiling on NDA downward (upward) by an amount equal to the surplus (shortfall) in actual net external assistance compared to expected net external assistance. For the purpose of calculating the adjustment for NDA, the amount of net external assistance will be converted into Guinean francs at the program exchange rate. For a shortfall in net external assistance, the total downward adjustment of NIR and the total upward adjustment of NDA will be capped at the equivalent of US\$20 million at the program exchange rate. 
Table 1. Guinea: Data Reporting Requirements

\begin{tabular}{|c|c|c|c|}
\hline $\begin{array}{l}\text { Category of } \\
\text { Data }\end{array}$ & Table/Report & Frequency & Deadline \\
\hline \multirow[t]{5}{*}{$\begin{array}{l}\text { Financial and } \\
\text { monetary data }\end{array}$} & $\begin{array}{l}\text { Central bank balance sheet, consolidated balance sheet of } \\
\text { commercial banks, monetary survey (at both the current and } \\
\text { program exchange rate) }\end{array}$ & Monthly & 30th of the month for the previous month \\
\hline & $\begin{array}{l}\text { Detailed net treasury position (NTP) and net government } \\
\text { position (NGP) }\end{array}$ & Monthly & $30^{\text {th }}$ of the month for the previous month \\
\hline & $\begin{array}{l}\text { Interest rates and outstanding volumes of government and } \\
\text { central bank securities (Bons Du Trésor and Titres de } \\
\text { Régulation Monétaire) }\end{array}$ & Monthly & $30^{\text {th }}$ of the month for the previous month \\
\hline & Prudential indicators of commercial banks & Quarterly & One month after end of quarter \\
\hline & Foreign exchange budget (budget en devises) & Monthly & $30^{\text {th }}$ of the month for the previous month \\
\hline \multirow[t]{8}{*}{ Fiscal data } & $\begin{array}{l}\text { Table of indicators (tableau de bord), including a detailed } \\
\text { statement of revenue, expenditure, and cash-flow operations }\end{array}$ & Monthly & $30^{\text {th }}$ of the month for the previous month \\
\hline & Treasury balances (balances générales du Trésor) & Monthly & $30^{\text {th }}$ of the month for the previous month \\
\hline & Cash-flow plan (plan de trésorerie) & Monthly & $30^{\text {th }}$ of the month for the previous month \\
\hline & Monthly government flow of funds table (TOFE) & Monthly & $30^{\text {th }}$ of the month for the previous month \\
\hline & $\begin{array}{l}\text { Expenditure reports by ministries in expenditure circuit } \\
\text { (chaîne des dépenses) }\end{array}$ & Monthly & $30^{\text {th }}$ of the month for the previous month \\
\hline & $\begin{array}{l}\text { Execution of budgetary expenditure, HIPC resources, and } \\
\text { other priority expenditure }\end{array}$ & Monthly & $30^{\text {th }}$ of the month for the previous month \\
\hline & $\begin{array}{l}\text { Outstanding Treasury float, including reimbursements of } \\
\text { VAT credits, and arrears on domestic debt recognized by the } \\
\text { government }\end{array}$ & Monthly & $30^{\text {th }}$ of the month for the previous month \\
\hline & $\begin{array}{l}\text { Nonbank financing, indicating transactions in Guinean francs } \\
\text { and those in foreign currencies. }\end{array}$ & Monthly & $30^{\text {th }}$ of the month for the previous month \\
\hline \multirow[t]{2}{*}{ Real sector data } & Consumer price index, Conakry & Monthly & $30^{\text {th }}$ of the month for the previous month \\
\hline & National accounts & Annual & $\begin{array}{l}\text { Summary estimates: three months after the } \\
\text { end of year }\end{array}$ \\
\hline \multirow{3}{*}{$\begin{array}{l}\text { Balance of } \\
\text { payments data }\end{array}$} & Imports by use and exports by major product, trade balance & Monthly & Three months after end of quarter \\
\hline & Price and volume indices of imports and of exports & Quarterly & Three months after end of quarter \\
\hline & Consolidated estimates of the balance of payments & Annual & $\begin{array}{l}\text { Summary estimates: six months after the } \\
\text { end of year }\end{array}$ \\
\hline \multirow[t]{5}{*}{ External debt } & Debt service due before and after debt relief & Monthly & $30^{\text {th }}$ of the month for the previous month \\
\hline & Debt service paid & Monthly & 30th of the month for the previous month \\
\hline & Debt service reconciliation table & Monthly & 30th of the month for the previous month \\
\hline & $\begin{array}{l}\text { Stock of outstanding debt at the end of the month, and daily } \\
\text { stock of debt service due (after relief) and not paid; daily } \\
\text { stock of arrears according to the program definition for } \\
\text { arrears. }\end{array}$ & Monthly & 30th of the month for the previous month \\
\hline & Drawings on new loans & Monthly & 30th of the month for the previous month \\
\hline \multirow[t]{2}{*}{$\begin{array}{l}\text { External grants } \\
\text { and loans }\end{array}$} & Disbursements & Quarterly & $\begin{array}{l}30^{\text {th }} \text { of the last month of the quarter for the } \\
\text { preceding quarter }\end{array}$ \\
\hline & $\begin{array}{l}\text { Monthly transfers of amounts of HIPC Initiative debt relief, by } \\
\text { creditor }\end{array}$ & Monthly & 30th of the month for the previous month \\
\hline
\end{tabular}




\section{Annex I. Guinea: Relations With The Fund \\ (As of May 31, 2008)}

I. Membership Status: Joined: September 28, 1963; Article VIII (obligations of Sections 2, 3, and 4 accepted on November 17, 1995.

II. General resources account:

Quota

Fund holdings of currency

Reserve Position

Holdings Exchange Rate

III. SDR department:

Net cumulative allocation

Holdings

IV. Outstanding purchases and loans:

Poverty Reduction and Growth Facility (PRGF) arrangements

$\begin{array}{rr}\text { SDR Million } & \text { \%Quota } \\ 107.10 & 100.00 \\ 107.03 & 99.93 \\ 0.08 & 0.07\end{array}$

SDR Million

17.60

0.29

SDR Million

35.21
\%Allocation

100.00

1.64

\%Quota

32.88

V. Financial Arrangements:

Date of

$\underline{\text { Type }}$

PRGF

PRGF $^{1}$

ESAF/PRGF
Arrangement

Dec 21, 2007

May 02, 2001

Jan 13, 1997
Expiration

Date

Dec 20, 2010

May 01, 2004

Jan 12, 2001
Amount Approved

(SDR Million)

48.20

64.26

70.80
Amount Drawn

(SDR Million)

6.89

25.70

62.94

VI. Projected payments to Fund (without HIPC assistance)

(SDR Million; based on existing use of resources and present holdings of SDRs):

Forthcoming

Principal

Charges/Interest

Total

\begin{tabular}{|c|c|c|c|c|}
\hline 2008 & 2009 & 2010 & 2011 & 2012 \\
\hline 6.11 & 8.29 & 7.50 & 3.86 & 2.57 \\
\hline 0.43 & 0.64 & 0.60 & 0.57 & 0.55 \\
\hline 6.54 & 8.93 & 8.10 & 4.43 & 3.12 \\
\hline
\end{tabular}

\footnotetext{
${ }^{1}$ The PRFG arrangement went off track as of December 2002.
} 
VII. Implementation of HIPC initiative:

Enhanced

A. Commitment of HIPC assistance

Framework

Decision point date

Dec 2000

Assistance committed

by all creditors (US\$ million) ${ }^{12}$

Of which: IMF assistance (US\$ million)

(SDR equivalent in millions)

Completion point date

Floating

B. Disbursement of IMF assistance (SDR Million)

Assistance disbursed to the member

10.02

Interim assistance

Completion point balance

Additional disbursement of interest income ${ }^{13}$

Total disbursements

10.02

\section{Safeguards assessments:}

Pursuant to Fund policy, the Central Bank of the Republic of Guinea (BCRG) was subject to an updated safeguards assessment in conjunction with a new PRGF arrangement. The safeguards assessment report that was completed on October 21, 2007, confirmed the continued existence of critical vulnerabilities in the BCRG safeguards framework, which would pose a high risk to the proper safeguarding of IMF disbursements. Overall, a system of strong internal controls, financial governance, and accountability needs to be urgently re-established and maintained at the BCRG. The assessment recommends measures that should help mitigate the safeguards risks, though significant risks would still remain. The BCRG has implemented critically important measures as prior actions for the PRGF. Several other recommendations are performance criteria and structural benchmarks for the program, and staff monitors their implementation as part of program conditionality.

\footnotetext{
${ }^{12}$ Assistance committed under the original framework is expressed in net present value (NPV) terms at the completion point, and assistance committed under the enhanced framework is expressed in NPV terms at the decision point. Hence these two amounts can not be added.

${ }^{13}$ Under the enhanced framework, an additional disbursement is made at the completion point corresponding to interest income earned on the amount committed at the decision point but not disbursed during the interim period.
} 


\section{Exchange arrangements:}

The regime is classified as a float. As of March 2006, the central bank calculates the official reference exchange rate daily as the weighted average rate of foreign exchange transactions in the banking sector and authorized foreign exchange bureaus (though the latter were excluded from the calculation starting in September 2007). Guinea continues to have a multiple currency practice arising from the absence of a mechanism to prevent a potential deviation of the exchange rate used by the BCRG in its foreign exchange transactions from the exchange rates used by the commercial banks in transactions with their customers. The official reference rate is applied only for accounting purposes.

\section{Article IV consultation:}

Guinea is currently on the 24-month consultation cycle. The last consultation was concluded by the Executive Board on December 21, 2007 (Country Report No. 08/33).

\section{Technical assistance provided in 2004-08:}

\section{A. AFRITAC West}

\begin{tabular}{|c|c|c|}
\hline Area & Focus & Timing \\
\hline $\begin{array}{l}\text { Public finance } \\
\text { management }\end{array}$ & Accounting reforms, capacity of the Treasury Directorate. & Mar. 2004 \\
\hline Tax administration & Modernization of tax administration. & Mar. 2004 \\
\hline Customs administration & Customs procedures. & May 2004 \\
\hline Public debt & Information system and public debt management. & Jun. 2004 \\
\hline $\begin{array}{l}\text { Tax and customs } \\
\text { administration }\end{array}$ & Tax and customs fraud (regional workshop). & Jun. 2004 \\
\hline Tax administration & Large taxpayer file, fiscal audits, and computerization of the DNI. & Dec. 2004 \\
\hline Public debt & Domestic debt management. & Jan. 2005 \\
\hline $\begin{array}{l}\text { Public finance } \\
\text { management }\end{array}$ & Budget and accounting management. & Jan.-Feb. 2005 \\
\hline Macroeconomic statistics & Public finance statistics (incl. implementation of GDDS). & Feb.-Mar. 2005 \\
\hline Microfinance & Microfinance regulation. & Mar. 2005 \\
\hline Tax administration & Unique taxpayer identifier. & Mar.-Apr. 2005 \\
\hline Microfinance & Microfinance regulation. & May 2005 \\
\hline Customs administration & Customs valuation. & Jun. 2005 \\
\hline
\end{tabular}




\begin{tabular}{|c|c|c|}
\hline Area & Focus & Timing \\
\hline Debt & Domestic debt management. & Jun. 2005 \\
\hline Microfinance & Microfinance regulation. & Aug. 2005 \\
\hline Microfinance & Technical support to the micro finance unit. & Sep.. 2005 \\
\hline Tax administration & Taxpayer file. & Oct. 2005 \\
\hline $\begin{array}{l}\text { Public finance } \\
\text { management }\end{array}$ & Implementing the accounting framework; new accounting plan. & Nov. 2005 \\
\hline Microfinance & Inspections. & Nov.-Dec. 2005 \\
\hline Microfinance & Inspections. & Jan.-Feb. 2006 \\
\hline Customs administration & $\begin{array}{l}\text { Controls on the value of imports, customs procedures compatible } \\
\text { with Sydonia }++ \text {. }\end{array}$ & Jan. 2006 \\
\hline Real sector statistics & Work plan to improve real sector statistics. & Feb. 2006 \\
\hline Tax administration & $\begin{array}{l}\text { Managing large, medium, and small taxpayers; tax audits; the } \\
\text { unique taxpayer number; and VAT credits. }\end{array}$ & Feb. 2006 \\
\hline $\begin{array}{l}\text { Public finance } \\
\text { management }\end{array}$ & Accounting framework. & Mar. 2006 \\
\hline Macroeconomic statistics & $\begin{array}{l}\text { Institutional environment for producing the table of financial } \\
\text { operations of the government (TOFE). }\end{array}$ & Apr. 2006 \\
\hline $\begin{array}{l}\text { Public finance } \\
\text { management }\end{array}$ & $\begin{array}{l}\text { Quality of the table of financial operations of the government } \\
\text { (TOFE) and its reconciliation with the net credit position of the } \\
\text { government (PNG). }\end{array}$ & Apr. 2006 \\
\hline Microfinance & System for authorization requests. & May 2006 \\
\hline Real sector statistics & Implementing the 1993 SNA. & May 2006 \\
\hline $\begin{array}{l}\text { Public finance } \\
\text { management }\end{array}$ & Follow up on recommendations of the Feb. 2005 FAD mission. & Jul. 2006 \\
\hline Real sector statistics & CPI index. & Jul.-Aug. 2006 \\
\hline Customs administration & $\begin{array}{l}\text { Follow up on } 2004 \text { FAD mission and 2005/06 AFRITAC West } \\
\text { missions. }\end{array}$ & Sep. 2006 \\
\hline Debt & Public debt yield (regional workshop). & Sep. 2006 \\
\hline Debt & Domestic debt management; management capacity. & Sep.-Oct. 2006 \\
\hline Real sector statistics & Implementing the 1993 SNA. & Sep.-Oct. 2006 \\
\hline Macroeconomic statistics & $\begin{array}{l}\text { Metadata for public finance statistics, } 2006 \text { IMF Manual of Public } \\
\text { Finance Statistics. }\end{array}$ & Oct. 2006 \\
\hline Tax administration & Modernizing tax administration. & Nov. 2006 \\
\hline Microfinance & Build capacity at the central bank, project FIRST. & Dec. 2006 \\
\hline $\begin{array}{l}\text { Public finance } \\
\text { management }\end{array}$ & Expenditure procedures, monitoring of payment arrears. & Jan.-Feb. 2007 \\
\hline $\begin{array}{l}\text { Public finance } \\
\text { management }\end{array}$ & Net credit position of the Treasury (PNT); satellite accounts. & Mar. 2007 \\
\hline Real sector statistics & Implementing the 1993 SNA. & Jun. 2007 \\
\hline
\end{tabular}




\begin{tabular}{|c|c|c|}
\hline Area & Focus & Timing \\
\hline Microfinance & Desk audits. & Jul. 2007 \\
\hline Real sector statistics & Preliminary national accounts for $2003-05$. & Jul. 2007 \\
\hline $\begin{array}{l}\text { Tax and customs } \\
\text { administration }\end{array}$ & Internal controls in revenue administration (regional workshop). & Jul. 2007 \\
\hline Tax administration & $\begin{array}{l}\text { Progress in implementing the Dec. } 2006 \text { action plan; assess TA } \\
\text { needs. }\end{array}$ & Jul. 2007 \\
\hline Debt & $\begin{array}{l}\text { Debt database, debt of the government vis-à-vis the central bank, } \\
\text { procedural guidance for domestic debt. }\end{array}$ & Jul.-Aug. 2007 \\
\hline $\begin{array}{l}\text { Public finance } \\
\text { management }\end{array}$ & Follow up on Jul. 2006 mission, notably for budget classification. & Aug. 2007 \\
\hline Customs administration & Computerization of customs procedures. & Aug. 2007 \\
\hline Microfinance & Analysis and desk audits. & Oct. 2007 \\
\hline Microfinance & Inspections. & Nov.-Dec. 2007 \\
\hline $\begin{array}{l}\text { Tax and customs } \\
\text { administration }\end{array}$ & Improving revenue administration (joint with FAD mission). & Nov.-Dec. 2007 \\
\hline Macroeconomic statistics & $\begin{array}{l}\text { Interrelations among macroeconomic accounts; implementing the } \\
2001 \text { Manual on Public Finance Statistics. }\end{array}$ & Jan.-Feb. 2008 \\
\hline Real sector statistics & Implementing the 1993 SNA. & Jan.-Feb. 2008 \\
\hline Debt & Statistical bulletin on external debt. & Feb. 2008 \\
\hline Real sector statistics & CPI unit training; quality of CPI compilation. & Mar.-Apr. 2008 \\
\hline
\end{tabular}

\section{B. Headquarters}

\section{Department Purpose}

\section{FAD}

FAD

FAD

FAD

FAD

FAD

MCM

MCM

$\mathrm{MCM}$ supervision
Advise on tax administration.

Review progress in implementing tariff and customs administration reforms and advise on a strategy for the next steps.

Advise on budget and accounting procedures and on government financial information systems.

Advise on tax administration reforms.

Advise on public finance management.

Advise on improving revenue administration

Advise on bank liquidity management, exchange operations, and

Advise on improving prudential instruments and upgrading the capacity of the banking supervision department.

Advise on an operational framework for monetary policy and bank liquidity management.

\section{Timing}

Jun. 2004

Dec. 2004

Feb. 2005

Apr. 2006, Mar. 2007

Aug. 2007

Nov. 2007

Mar. 2004

Follow-up: Sep. 2004

Mar. 2005

Mar.-Apr. 2005 


\begin{tabular}{lll}
\hline Department & Purpose & Timing \\
\hline MCM & Follow-up mission on banking supervision. & May 2005 \\
MCM & Technical assistance needs assessment mission to the BCRG. & Jun. 2005 \\
MCM & Advise on monetary and exchange operations and supervision. & Aug. 2005 \\
MCM & Formulate a draft law on money laundering prevention. & Jan.-Feb. 2006 \\
MCM & Capacity-building in foreign exchange and monetary operations and & Oct. 2006 \\
& supervision. & Oct. 2006 \\
MCM & Assess the BCRG information and communications environment. & Jun.-Jul. 2007 \\
MCM & Technical assistance needs assessment mission to the BCRG. & Aug.-Sep. 2007 \\
MCM & Advise on foreign exchange operations and market development. & Dec. 2007 \\
MCM & Advise on monetary operations and liquidity management & Nov. 2007 \\
STA & Improve data collection, compilation, and dissemination practices for & \\
& monetary and financial statistics. & Jan. 2008 \\
\hline
\end{tabular}

\section{Resident representative:}

Mr. Alvin Hilaire took up his position as Resident Representative in November 2006. 


\section{AnNex II. Guinea: BANK-Fund COLlaboration \\ (As of June 18, 2008)}

\begin{tabular}{llcl}
\hline Title & Products & $\begin{array}{c}\text { Provisional Timing } \\
\text { of Missions } \\
\text { (if relevant) }\end{array}$ & $\begin{array}{c}\text { Expected } \\
\text { Delivery Date }\end{array}$ \\
\hline
\end{tabular}

\section{A. Mutual Information on Relevant Work Programs}

Bank work program in next 12 months

IMF work program in next 12 months

Fund request to Bank
- Interim Strategy Note.

- $\quad$ Emergency Support.

Sep. 2008

- Public Expenditure Review in education.

- ESW on agriculture growth.

- TA on public financial management.

- $\quad$ Advice on payroll management and incentive system for employees in the education sector.

- $\quad$ TA on Telecom Regulation (IDF).

- $\quad$ TA for investment framework reforms in the mining and non-mining sector.

- $\quad$ Capacity building for mining revenues.

- $\quad$ Advice and support for EITI 2006 implementation.

- Advice and support for EITI 2007 implementation.

- $\quad$ PSIA on the impact of food and oil price increases.

- Electricity Tariff Study.

- Water and electricity sector efficiency project.

- $\quad$ First review of the PRGF.

- $\quad$ Second review of the PRGF.

- $\quad$ HIPC completion point review.

- $\quad$ Inputs on bilateral debt for HIPC DSA.

- Analytical work in support of private sector policies (ICA).

- Technical assistance on PFM Action Plan.

\section{B. Request for Work Program Inputs}

- Review of 2007 public expenditures in education assistance to the elaboration of sector MTEFs and monitoring of spending allocations in 2008 budget and the 2009 draft budget.

- $\quad$ TA on designing a wage incentive system in civil service.
Jul. 2008

Nov. 2008

Aug. 2008

Oct. 2008; Feb. 2009

Jun. 2009

Sep. 2008

Dec. 2009

Apr. 2008; Jun. 2008

Nov. 2008

Oct. 2009

Nov. 2011

Sep. 2008

Dec. 2009

Sep. 2008

Dec. 2009

Ongoing

Nov. 2008

May 2008

May. 2009

Mar. 2008

Apr. 2008

Jun. 2008

Jan. 2009

Sep. 2008

Dec. 2012

Mar. 2008

Jul. 2008

Sep. 2008

Dec. 2008

Sep. 2008

Dec. 2008

Jul. 2008

Sep. 2008

Mar. 2008

Dec. 2009

Jul. 2008

Dec. 2009
Jul. 2008

Apr. 2008; Jun. 2008
Nov. 2008 


\begin{tabular}{|c|c|c|c|}
\hline Title & Products & $\begin{array}{l}\text { Provisional Timing } \\
\text { of Missions } \\
\text { (if relevant) }\end{array}$ & $\begin{array}{l}\text { Expected } \\
\text { Delivery Date }\end{array}$ \\
\hline \multirow{3}{*}{$\begin{array}{l}\text { Bank request to } \\
\text { Fund }\end{array}$} & $\begin{array}{l}\text { - Advice on designing measures to mitigate the } \\
\text { impact of food and oil price increases on the poor } \\
\text { and building capacity. }\end{array}$ & Ongoing & \multirow{4}{*}{ Sep.2008 } \\
\hline & $\begin{array}{l}\text { Review of BCRG operations and governance } \\
\text { adequacy. }\end{array}$ & Ongoing & \\
\hline & - TA on tax revenue management. & Ongoing & \\
\hline \multirow{5}{*}{$\begin{array}{l}\text { Joint products in } \\
\text { next } 12 \text { months }\end{array}$} & \multicolumn{2}{|c|}{ C. Agreement on Joint Products and Missions } & \\
\hline & - $\quad$ HIPC DSA for completion point. & & Nov. 2008 \\
\hline & - Joint mission on mining taxation issues. & Sept./Oct. 2008 & Dec. 2008 \\
\hline & - JSAN & Sept./Oct. 2008 & Dec. 2008 \\
\hline & - $\quad$ FSAP & May 2009 & May 2010 \\
\hline
\end{tabular}




\section{AnNeX III. Guinea: Statistical IsSUeS}

1. Data provision has serious shortcomings that significantly hamper surveillance, with staff's analysis affected particularly by shortcomings in balance of payments statistics and inconsistencies between fiscal data and monetary accounts. In addition, changes in the financial system in recent years have adversely affected the quality of data for deposit money banks.

2. Although a law on statistics that organizes the institutional setting for producing statistics at a decentralized level was approved in 1995, neither the National Direction of Statistics (DNS), responsible for coordinating the production of statistics, nor a technical committee responsible for providing recommendations on the statistical program - both created by the new law - seem to have improved the quality of statistics.

3. The country participates in the General Data Dissemination System (GDDS). However, plans for improvements and metadata for all data categories need to be updated.

\section{Real sector statistics}

4. Real sector statistics are weak, incomplete, and published with insufficient timeliness to support economic policy making. Only the monthly consumer price index (CPI) is published in a timely manner; other statistics are published less frequently and less regularly, and are often not reliable. Some of the series are available on the DNS website. ${ }^{14}$ Monthly surveys of mining, industrial and agricultural production are produced with long delays. Employment and population statistics are only published annually. A program to reinforce the national accounts is being undertaken with the assistance of the regional technical assistance center (West AFRITAC) and the Gesellschaft für Technische Zusammenarbeit. The work is expected to lead to the production of annual input-output matrices. A recent mission by West AFRITAC helped the authorities prepare final estimates for the new base year-2003, complete the provisional national accounts estimates for the period 2003-05, and advance compilation of provisional estimates for 2006, whose release is expected shortly.

5. A monthly CPI for Conakry is available, with a one-month lag. Export prices are estimated on the basis of information supplied directly by the mining companies, and import prices are based on a weighted average of partner country export prices. Exchange rates are reported daily.

6. Additional data on the agriculture sector have been published sporadically in the form of Food and Agriculture Organization/United Nations Development Program surveys. No data on employment or labor costs are published.

\footnotetext{
${ }^{14}$ See http://www.afristat.org/ins-guinee/index.htm.
} 


\section{Balance of payments statistics}

7. A March 2008 mission found that the presentation of the balance of payments statement is in conformity with BPM5 recommendations. However, the quality of data is poor, mainly because of insufficient work input at the BCRG, and defective work coordination with other institutions involved in the collection of data. In particular, the Technical Committee on Balance of Payments Harmonization has ceased its activities. In this context, almost all items of the balance of payments need improvements, in particular in the current account.

8. The mission identified three main issues, to be treated as priority: (i) trade statistics are collected through customs and processed by the Eurotrace software, which need to be adjusted for coverage and valuation (one conclusion of the mission is that exports are probably underestimated); (ii) absence of direct investment transactions, mainly because the questionnaires are not well designed, and also the response rate is poor (as a result, mining companies' financial transactions and investment income are not reflected in the BOP); (iii) complete lack of data on exchange of foreign banknotes, a considerable local activity. This is a strong impediment for assessing some transactions in the BOP, in particular workers remittances.

9. In this context, the most urgent tasks for the BCRG are: (i) to reinforce staff resources for the balance of payments unit; (ii) to restore the activities of the Technical Committee on Balance of Payments Harmonization (which should also include the Customs Directorate and the Ministry of Mining and Geology), with the priority task to improve the quality of trade statistics; (iii) to launch a revised questionnaire (designed by the mission) to mining companies; and (iv) to find ways to better assess the amounts involved in exchange of foreign banknotes, possibly through a survey of bureau de change. In addition, the mission made recommendations to improve several items of the balance of payments, in particular freight, travel and government services, external debt statistics, and inclusion of BIS data on foreign assets of the private sector.

10. The BCRG last reported balance of payments data to STA in 2006 (data for the years 2003-04).

\section{Government finance statistics}

11. The Ministry of Finance, Economy and Planning compiles comprehensive monthly budgetary central government data on a cash basis for revenue and on commitment and cash bases for expenditure. AFR receives preliminary data within one month. Budgetary data are often not internally consistent.

12. The budget includes the bulk of all government operations, although it excludes a number of "satellite" accounts that are not directly incorporated into the budget. There are also significant differences between the national definition of general government and that provided in the Government Finance Statistics Manual 2001 (GFSM 2001). Moreover, autonomous funds, such as the Road Fund, are only partly incorporated into the budget. For the Road Fund, 
the largest autonomous fund, 100 percent of resources are "committed" through the budget and transferred from the budget to the fund. Actual disbursements are made at the discretion of the Road Fund (though monitored through its account at the central bank). The fuel tax is not earmarked for the Road Fund, but it is received by the general budget and transferred to the Road Fund through the budget.

13. A January 2008 STA mission assisted the authorities in preparing fiscal data using the GFSM 2001 analytical framework. In particular, the mission: (i) assessed institutional coverage and data sources of the data reported in the TOFE; (ii) refined and supplemented the tables that bridge the national classification to the GFSM 2001 framework; and (iii) assisted the authorities with the preparation of the annual and high frequency GFS for publication in the IMF GFS Yearbook and International Financial Statistics. The latest data published in the GFS Yearbook are for 1999 and are on a cash basis only. Guinea does not report fiscal data for publication in International Financial Statistics (IFS).

\section{Monetary accounts statistics}

14. Developments in the financial system over the last few years, including the liquidation and restructuring of a number of banks, are not fully reflected in the monetary statistics, partly because of problems in collecting data. STA recently fielded a follow-up monetary and financial mission during November 19-30, 2007. The mission (i) provided advice to improve data collection, compilation, and dissemination practices; (ii) provided guidance for the migration to the standardized report forms and (iii) updated the GDDS metadata for the financial sector. The mission also followed-up on issues raised by AFR, such as the procedures for monthly reporting of the national currency in circulation and consistency of the central bank's balance sheet data with the external auditor's recommendations. A work program (with timetable) for implementing the mission's recommendations was agreed with the BCRG.

15. Monthly data on monetary authorities, deposit money banks, and interest rates are provided to AFR with a one-month delay; however, the most recent monetary data transmitted to STA for publication in IFS are for September 2006. 


\section{Guinea: Table of Common Indicators Required for Surveillance}

(As of June 17, 2008)

\begin{tabular}{|c|c|c|c|c|c|}
\hline & $\begin{array}{l}\text { Date of } \\
\text { latest } \\
\text { observation }\end{array}$ & $\begin{array}{l}\text { Date } \\
\text { received }\end{array}$ & $\begin{array}{l}\text { Frequency } \\
\text { of Data }^{6}\end{array}$ & $\begin{array}{l}\text { Frequency } \\
\text { of Reporting }\end{array}$ & $\begin{array}{l}\text { Frequency } \\
\text { of publication }\end{array}$ \\
\hline Exchange Rates & $6 / 10 / 08$ & $6 / 10 / 08$ & $\mathrm{D}$ & $\mathrm{D}$ & $\mathrm{D}$ \\
\hline $\begin{array}{l}\text { International Reserve Assets and Reserve } \\
\text { Liabilities of the Monetary Authorities }{ }^{1}\end{array}$ & $5 / 30 / 08$ & $6 / 12 / 08$ & M & M & M \\
\hline Reserve/Base Money & $4 / 30 / 08$ & $6 / 12 / 08$ & M & M & M \\
\hline Broad Money & $4 / 30 / 08$ & $6 / 12 / 08$ & M & M & M \\
\hline Central Bank Balance Sheet & $4 / 30 / 08$ & $6 / 12 / 08$ & M & M & M \\
\hline $\begin{array}{l}\text { Consolidated Balance Sheet of the Banking } \\
\text { System }\end{array}$ & $4 / 30 / 08$ & $6 / 12 / 08$ & M & M & M \\
\hline Interest Rates ${ }^{2}$ & $4 / 30 / 08$ & $6 / 12 / 08$ & M & M & M \\
\hline Consumer Price Index & $04 / 30 / 2008$ & $5 / 23 / 08$ & M & M & M \\
\hline $\begin{array}{l}\text { Revenue, Expenditure, Balance and } \\
\text { Composition of Financing }{ }^{3}-\text { General } \\
\text { Government }^{4}\end{array}$ & NA & --- & --- & --- & --- \\
\hline $\begin{array}{l}\text { Revenue, Expenditure, Balance and } \\
\text { Composition of Financing }{ }^{3}-\text { Central } \\
\text { Government }\end{array}$ & $04 / 30 / 08$ & $6 / 11 / 08$ & M & M & M \\
\hline $\begin{array}{l}\text { Stocks of Central Government and Central } \\
\text { Government-Guaranteed Debt }{ }^{5}\end{array}$ & 2007 & $3 / 21 / 08$ & A & A & A \\
\hline External Current Account Balance & 2006 & $4 / 25 / 07$ & A & A & A \\
\hline Exports and Imports of Goods and Services & 2006 & $4 / 25 / 07$ & A & A & A \\
\hline GDP/GNP & 2006 & $4 / 25 / 07$ & A & A & A \\
\hline Gross External Debt & 2006 & $4 / 25 / 07$ & A & A & A \\
\hline
\end{tabular}

1 Includes reserve assets pledged or otherwise encumbered as well as net derivative positions.

2 Both market-based and officially-determined, including discount rates, money market rates, rates on treasury bills, notes and bonds.

3 Foreign, domestic bank, and domestic nonbank financing.

4 The general government consists of the central government (budgetary funds, extra budgetary funds, and social security funds) and state and local governments.

5 Including currency and maturity composition.

6 Daily (D), Weekly (W), Monthly (M), Quarterly (Q), Annually (A), Not Available (NA). 


\section{Statement by the IMF Staff Representative \\ July 28, 2008}

1. This statement reports on developments, including the status of the two prior actions, since finalizing the staff report for the first review under the three-year arrangement under the Poverty Reduction and Growth Facility (PRGF), request for waiver of nonobservance of performance criteria, modification of performance criteria, augmentation of access, and financing assurances review. The thrust of the staff appraisal has not changed.

2. The authorities have implemented both prior actions for the first review. On June 27 th the Council of Ministers adopted the multiyear strategic plan for public finance management. The plan was disseminated to key donors, who met to coordinate support for its implementation. Also on June 27th, the Council of Ministers approved an emergency plan for the electricity company EDG. The plan focuses on improving collection rates - including by installing meters - and combating fraud. In addition, electricity tariffs for large users were raised between 63 and 74 percent on July 14 (effective May 1st, 2008), the first such increase since July 2006.

\section{Available information as of end-May 2008, suggests that mid-year program fiscal and} monetary targets are within reach. Government revenues are recovering from lows in the first quarter, reflecting mostly good performance in the mining sector, and spending remains under control. The central bank has tightened monetary policy, following higher-than-projected increases in monetary aggregates in the first quarter of 2008. Overdrafts of public enterprises in the central bank's books were kept below the program ceiling. However, the twelve-month inflation rate rose to 25.1 percent in May on account of higher food and oil prices. The exchange rate has been hovering around 4,500 GNF per U.S. dollar since end-April 2008, following a depreciation of about 8 percent in the first four months of the year.

4. The Guinean authorities continue to engage their external creditors. Since the Paris Club (PC) agreement of January 2008, they have signed bilateral agreements with six PC members (Austria, Belgium, France, Italy, Norway, and the USA), and efforts are underway with the remaining ones. The authorities are also discussing with Saudi Arabia and Kuwait a debt agreement on comparable terms, and have tried to engage in similar discussions with other non-PC creditors with the assistance of the PC Secretariat.

5. The independent external auditor has certified the central bank's 2007 financial statements, albeit with qualifications, noting improvements in accounting and control procedures. This is a major improvement from the adverse opinion expressed last year on the bank's 2006 financial statements. 
Press Release No. 08/185

FOR IMMEDIATE RELEASE

July 28, 2008
International Monetary Fund

Washington, D.C. 20431 USA

\section{IMF Executive Board Completes First Review under PRGF Arrangement for Guinea, Increases Financial Assistance to Mitigate Food and Fuels Price Impact, and Approves US\$28.7 Million Disbursement}

The Executive Board of the International Monetary Fund (IMF) today completed the first review of Guinea's performance under a three-year Poverty Reduction and Growth Facility (PRGF) arrangement. The Board also approved an SDR 21.42 million (about US\$34.9 million) augmentation in the arrangement to help Guinea cope with the recent external shocks from rising food and fuel prices. The completion of the review enables the disbursement of SDR 17.595 million (about US\$28.7 million), which would bring total disbursements under the arrangement to SDR 24.48 million (about US\$39.9 million), including part of the augmented amount.

The Executive Board also granted waivers of non-observance for two performance criteria related to net international reserves and external payments arrears, on the basis of remedial actions taken, and decided to modify the performance criteria for the next disbursement in view of the adverse external shock that Guinea is experiencing. The Board completed the country's financing assurances review under the arrangement as well.

The three-year PRGF arrangement for Guinea was originally approved by the Executive Board on December 21, 2007 (see Press Release No. 07/309) in an amount equivalent to SDR 48.195 million (about US\$78.5 million). With the Board's approval of the augmentation today, the total amount of the arrangement will be equivalent to SDR 69.615 million (about US\$113.4 million).

Following the Executive Board's discussion, Mr. Murilo Portugal, Deputy Managing Director and Acting Chair, said: 
"Guinea's economic stabilization policies were successful in 2007. Inflation fell significantly and the economy began to recover from social turmoil early in the year. The basic primary fiscal surplus improved, and the authorities refrained from recourse to central bank financing.

"The large international fuel and food price shock is creating serious challenges to the shortterm outlook. The government courageously raised local petroleum prices substantially early in the year and further measures to ensure the pass-through of world oil prices are planned for the period ahead. It will be important to target measures to alleviate the impact of higher food prices on the most vulnerable.

"To help absorb the effect of higher food and fuel prices on the balance of payments and accomplish the program objectives in 2008 , including the rebuilding of foreign reserves, well-coordinated economic policies, and the mobilization of additional external assistance will be needed. The Fund has augmented financial resources to Guinea's PRGF arrangement. This, in conjunction with other donors' support, will help in mitigating the impact of the surge in oil and food prices.

"A proactive monetary policy will help manage liquidity growth and contain inflationary pressures. Continued fiscal adjustment can be achieved through improving revenue collection and containing the budget's exposure to loss-making public enterprises. Non-priority expenditures need to be curtailed to create the fiscal space for increased pro-growth and propoor spending.

"It will be important to accelerate structural reforms to achieve sustained private sector- and foreign direct investment-led growth and reduce poverty. Efforts to improve the management of Guinea's abundant natural resources will help the country benefit from the worldwide boom in commodity prices. The plans to improve public financial management, and to reform the central bank and enhance its independence are also important priorities.

"Continued implementation of the PRGF-supported program will help pave the way for Guinea to achieve the Completion Point under the enhanced Heavily Indebted Poor Countries (HIPC) Initiative and benefit from the Multilateral Debt Relief Initiative (MDRI). It will be important for the authorities to strengthen foreign exchange and debt management to avoid the recurrence of external arrears, and safeguard debt sustainability," Mr. Portugal said.

The PRGF is the IMF's concessional facility for low-income countries. PRGF loans carry an annual interest rate of 0.5 percent and are repayable over 10 years with a $5 \frac{1}{2} 2$-year grace period on principal payments. 


\section{Statement by Laurean Rutayisire, Executive Director for Guinea July 28, 2008}

\section{Introduction}

I would like to express the deep appreciation of my Guinean authorities to Management and Staff for their support and assistance in implementing the PRGF arrangement. In December 2007 a three-year PRGF arrangement was put in place whose successful implementation is enabling the authorities to stabilize the economy as demonstrated by the restoration of macroeconomic stability, the return of confidence in the national currency and reversal of the trend in growth decline.

Despite a very difficult social and political environment, the authorities' strong commitment to the program has enabled them to meet most of the quantitative performance criteria set for end-December 2007. Only two criteria were not met. The criterion for accumulation of net international reserves was missed due to higher than expected public expenditure in foreign currencies at year-end. Also the criterion for non accumulation of external payment arrears to certain multilateral creditors was not satisfied due to inadequate level of international reserves. However, it is worth noting that the total amount of these arrears was paid at the end of March 2008. With regard to the structural reform agenda, all structural performance criteria and benchmarks for end-December 2007 were met. Some structural benchmarks for early 2008 have, however, been delayed. Notably, the authorities deemed indispensable to postpone to end-November 2008 the implementation of the monthly automatic oil price adjustment mechanism in order to avoid further social unrest. In particular, as a result of the impact of the food and oil price rises, the country experienced in May and June 2008 a severe socio political crisis which led to a new Prime Minister being appointed. The new Prime Minister formed a new government in which he retained the previous economic management team. The new Prime Minister has also reaffirmed his government's commitment to a determined implementation of the PRGF program.

Based on the demonstrated appreciable performance despite the very difficult socio economic environment, my Guinean authorities are requesting completion of the first review under the PRGF arrangement. They are also requesting waivers for the non observance of two quantitative performance criteria, modification in the quantitative performance criteria and for augmentation of access to PRGF resources.

\section{Recent Macroeconomic Developments and Program Performance}

Although adverse developments in international markets compounded by the impact of food and oil price shocks have exerted severe negative impacts on Guinean economy, a positive 
real GDP growth was recorded in 2007. The exchange rate premium on the parallel market declined and inflation rate fell below program target although the rate remained at double digits. The budget deficit and money supply growth were also kept within the program targets although the external position remained difficult.

On the fiscal front, program targets for 2007 were broadly achieved. Measures adopted to reinforce customs and tax administration coupled with cuts in non priority spending resulted in improvement of the basic primary fiscal balance exceeding the program target by 0.2 percent of GDP. The better fiscal performance achieved was in spite of the measures which the authorities adopted to cushion the population from the adverse impact of the food and oil price escalation, which involved raising allowances for civil servants and students, subsidies for school transportation, and suspension of import duties on rice.

As regards monetary policy, the Central Bank has continued implementing zero government financing policy which has brought monetary expansion under control. In 2007, increases in reserve money and broad money were kept below nominal GDP growth. Banking credit remained below projections due in particular to the enforcement of the capital-adequacy ratio by the Central Bank and the sluggishness in the credit market. In order to further reinforce the Central Bank capacity to conduct monetary policy, the management and governance of the central bank have been enhanced in compliance with the recommendations made by external auditors and IMF's safeguards assessment mission.

In the structural reform area, the authorities also made significant progress. Audits of ministries have been completed and published. The 2005 report on government revenue from the mining sector was also published and the results of 2006 audit are expected by endNovember 2008. An investment committee was established at the central Bank to upgrade to international standards the surveillance, management and monitoring of international reserve accounts. In collaboration with donors, a multiyear strategy guiding public finance management reform has also been prepared and adopted. Trade restriction measures involving ban on exports of agricultural, forestry and fishing products were also lifted.

On debt sustainability, the authorities have initiated discussions with creditors in order to restructure the country's external debt. In January 2008, agreements with four Paris Club Member countries were signed. The authorities have also begun negotiations with non Paris Club creditors to seek debt restructuring in terms comparable with Paris Club.

\section{The 2008 Program Policies and Objectives}

My Guinean authorities are strongly determined to consolidate the gains achieved in macroeconomic management and speed up structural reforms in order to accelerate economic growth and reduce poverty.

In the real sector, my authorities' major priorities are to implement their new Poverty Reduction Strategy whose emphasis is on the development of agricultural sector, investment in infrastructure, education and health sectors. In this regard the authorities will pursue their 
efforts to increase productivity in the agricultural sector and investment in infrastructure to remove constraint on growth.

In the Fiscal Sector, the authorities' efforts aiming at improving fiscal management will be accelerated. Tax and Customs administration will be strengthened including controls on customs exemptions through the implementation of recommendations made by FAD technical mission including a quarterly monitoring of the execution of performance contracts approved by Tax and Customs administration. The authorities will continue to execute the budget without net monetary financing from the Central Bank. The authorities also plan to adopt the new budget classification and definition of poverty-reducing expenditures in the context of 2009 budget preparation. Fiscal exposure to public enterprises will also be contained.

In the Monetary and Financial Policies Area, the authorities will aim at containing inflation, increasing the level of international reserves and further improving financial intermediation and private sector access to credit. In this regard, the authorities intend to keep the money supply within the limits of the program through proactive management of bank liquidity and if necessary by improving the attractiveness for banks of monetary regulation securities. The flexible exchange rate regime will be preserved and the authorities will continue gradually to build up the international reserves in order to increase the import cover. In order to enhance the independence of the central bank and guarantee the transparency of its operations, the authorities will present to the Parliament by November 2008 a new central bank's law. Measures to further reinforce the banking supervision will also be pursued in order maintain the soundness of the financial sector.

On Structural Reforms, the authorities are committed to sustaining the implementation of their structural reform agenda. They will notably pursue efforts to create conducive environment for foreign direct investment in the country's natural resources. To this end, my authorities will adopt by end of 2008 a standard mining contract and revise mining taxation. Guinea has already adhered to EITI and agreed to be a pilot country for the World Banksponsored enhancing of the Initiative and to speed up the implementation and posting of EITI audits.. Given the recent shocks the country has experienced with regard to the increase of food prices, the authorities have begun to mobilize donors' support in providing farmers with better and sustained access to seeds, fertilizers and pesticides. My authorities call for further donor support in this area.

In order to strengthen the monitoring of the program and ensure the coordination at the highest level, a high level committee has been established to review program implementation including the payment of external debt.

The authorities are mindful on the need to improve the coverage and quality of statistical data for the monitoring of the program. They will continue to strengthen their efforts including the implementation of recommendations made by the recent IMF technical mission notably on balance of payments statistics. The authorities intend also to document the PRSP monitoring indicators and prepare the report on the first year of PRSP implementation. 


\section{Conclusion}

My Guinean authorities remain strongly committed to implementing prudent fiscal and monetary policies and structural reforms under the PRGF-arrangement. They are determined to continue working with the Fund and other development partners to sustain the implementation of their economic and financial program. Guinean authorities request Board approval of the completion of the first review under the PRGF-supported program, waiver for the program performance criteria missed, modifications in the quantitative performance criteria, and augmentation of access to PRGF resources. 